TRANSACTIONS OF THE

AMERICAN MATHEMATICAL SOCIETY

Volume 365, Number 8, August 2013, Pages 4121-4151

S 0002-9947(2013)05729-7

Article electronically published on March 28, 2013

\title{
A UNIFORM BIJECTION BETWEEN NONNESTING AND NONCROSSING PARTITIONS
}

\author{
DREW ARMSTRONG, CHRISTIAN STUMP, AND HUGH THOMAS
}

\begin{abstract}
In 2007, D.I. Panyushev defined a remarkable map on the set of nonnesting partitions (antichains in the root poset of a finite Weyl group). In this paper we use Panyushev's map, together with the well-known Kreweras complement, to construct a bijection between nonnesting and noncrossing partitions. Our map is defined uniformly for all root systems, using a recursion in which the map is assumed to be defined already for all parabolic subsystems. Unfortunately, the proof that our map is well defined, and is a bijection, is case-by-case, using a computer in the exceptional types. Fortunately, the proof involves new and interesting combinatorics in the classical types. As consequences, we prove several conjectural properties of the Panyushev map, and we prove two cyclic sieving phenomena conjectured by D. Bessis and V. Reiner.
\end{abstract}

\section{INTRODUCTION}

To begin we will describe the genesis of the paper.

1.1. Panyushev complementation. Let $\Delta \subseteq \Phi^{+} \subseteq \Phi$ be a triple of simple roots, positive roots, and a crystallographic root system corresponding to a finite Weyl group $W$ of rank $r$. We think of $\Phi^{+}$as a poset in the usual way, by setting $\alpha \leq \beta$ whenever $\beta-\alpha$ is in the nonnegative span of the simple roots $\Delta$. This is called the root poset. The set of nonnesting partitions $\mathrm{NN}(W)$ is defined to be the set of antichains (sets of pairwise-incomparable elements) in $\Phi^{+}$. This name is based on a pictorial representation of antichains in the classical types. It is well known that the number of nonnesting partitions is equal to the Catalan number

$$
\operatorname{Cat}(W):=\prod_{i=1}^{r} \frac{d_{i}+h}{d_{i}},
$$

where $d_{1} \leq d_{2} \leq \cdots \leq d_{r}=h$ are the degrees of a fundamental system of polynomial invariants for $W$ (called the degrees of $W$ ), and where $h$ is the Coxeter number. This formula was first conjectured by Postnikov [21, Remark 2] and at least two uniform proofs are known: Cellini and Papi [8] established a bijection

Received by the editors March 9, 2011 and, in revised form, October 7, 2011

2010 Mathematics Subject Classification. Primary 05A05; Secondary 20F55.

Key words and phrases. Weyl groups, Coxeter groups, noncrossing partitions, nonnesting partitions, cyclic sieving phenomenon, bijective combinatorics.

During the time that he worked on this paper, the first author was supported by NSF Postdoctoral Fellowship DMS-0603567 and NSF grant DMS-1001825.

The second author was supported by a CRM-ISM postdoctoral fellowship. He would like to thank the Fields Institute for its hospitality during the time he was working on this paper.

The third author was supported by an NSERC Discovery Grant. He would like to thank the Norges teknisk-naturvitenskapelige universitet and the Fields Institute for their hospitality during the time he was working on this paper. 


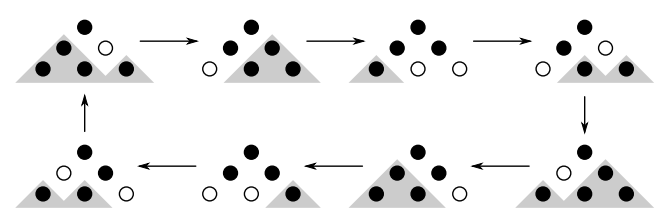

Figure 1. An orbit of the Panyushev complement.

between antichains in the root poset and dominant chambers of the Shi arrangement. The enumeration then follows from earlier work of Haiman 14 on the finite torus $Q /(h+1) Q$, or from subsequent work of Athanasiadis [2] on characteristic polynomials of hyperplane arrangements.

In 2007, Panyushev defined a remarkable map on nonnesting partitions [20]. To describe it, we first note that an antichain $I \subseteq \Phi^{+}$corresponds bijectively to the order ideal $\langle I\rangle \subseteq \Phi^{+}$that it generates. The Panyushev complement is defined as follows.

Definition 1.1. Given an antichain of positive roots $I \subseteq \Phi^{+}$, define $\operatorname{Pan}(I)$ to be the antichain of minimal roots in $\Phi^{+} \backslash\langle I\rangle$.

For example, Figure 1 displays a single orbit of the Panyushev complement acting on the root poset of type $A_{3}$. The antichain in each picture corresponds to the maximal black dots in the order ideal given by the shaded area.

We note that this action on antichains can be applied to an arbitrary poset, and, in fact, this operation has been rediscovered several times, going back at least to Duchet [10] for Boolean lattices and to Brouwer-Schrijver [7] for arbitrary posets. In this paper, we keep the expression "Panyushev complement" because Panyushev observed that this map on the root poset has some special properties not holding in an arbitrary poset. In particular, in [20], he made the following conjectures, which have remained open even in type $A$.

Panyushev Conjectures. Let $W$ be a finite Weyl group of rank $r$, with $h$ its Coxeter number, and let Pan be the Panyushev complement on antichains in the associated root poset $\Phi^{+}$. Moreover, let $\omega_{0}$ be the unique longest element in $W$.

(i) $\operatorname{Pan}^{2 h}$ is the identity map on $\mathrm{NN}(W)$.

(ii) $\mathrm{Pan}^{h}$ acts on $\mathrm{NN}(W)$ by the involution induced by $-\omega_{0}$.

(iii) For any orbit $\mathcal{O}$ of the Panyushev complement acting on $\mathrm{NN}(W)$, we have

$$
\frac{1}{|\mathcal{O}|} \sum_{I \in \mathcal{O}}|I|=r / 2 .
$$

For example, in type $A_{3}$ we have $2 h=8$, and the Panyushev complement has three orbits, of sizes 2,4 , and 8 (the one pictured). In type $A, \omega_{0}$ acts by $\alpha_{i} \mapsto$ $-\alpha_{n-i}$, where $\alpha_{i}$ denotes the $i$-th simple root in the linear ordering of the Dynkin diagram. One can observe in the pictured orbit that $\mathrm{Pan}^{h}$ acts by reflecting the root poset (this corresponds to reversing the linear order of the Dynkin diagram), and that $\operatorname{Pan}^{2 h}$ is the identity map. Moreover, the average size of an antichain in this orbit is $\frac{1}{8}(2+1+1+2+2+1+1+2)=3 / 2=r / 2$.

In this paper we will prove the following.

Theorem 1.2. The Panyushev Conjectures are true. 


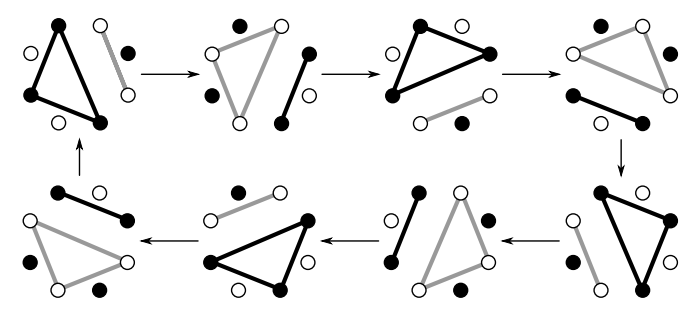

Figure 2. An orbit of the Kreweras map.

However, this theorem is not the main goal of the paper. Instead, we will use the Panyushev complement as inspiration to solve an earlier open problem: to construct a uniform bijection between antichains in $\Phi^{+}$and a different sort of Catalan object, the noncrossing partitions. We will then use the combinatorics we have developed to prove the Panyushev Conjectures.

1.2. Kreweras complementation. In addition to nonnesting partitions, there is also a notion of noncrossing partitions for root systems, which we now describe.

Let $T$ be the set of all reflections in a finite Coxeter group $W$ of rank $r$. That is, $T$ consists of the reflections orthogonal to the positive roots $\Phi^{+}$of a (not necessarily crystallographic) root system $\Phi$. Let $S \subseteq T$ denote the simple reflections, orthogonal to the simple roots $\Delta \subseteq \Phi^{+} \subseteq \Phi$, and let $c \in W$ be a Coxeter element (the product of the reflections $S$ in some order). Then the set of noncrossing partitions is

$$
\mathrm{NC}(W, c):=\left\{w \in W: \ell_{T}(w)+\ell_{T}\left(c w^{-1}\right)=r\right\} \subseteq W .
$$

For a full exposition of this object and its history, see [1]. It turns out that $\mathrm{NC}(W, c)$ is also counted by the Catalan number Cat $(W)$, but in this case no uniform proof is known (the only proof is case-by-case, using a computer for the exceptional types). In this paper we will partially remedy this situation by uniformly constructing a bijection between antichains in $\Phi^{+}$and $N C(W, c)$. It is only a partial remedy because the proof that our map actually is a bijection is still case-by-case.

Our map relies on the Panyushev complement and a certain map on noncrossing partitions, which we now describe. The type $A$ noncrossing partitions were first studied in detail by Kreweras [19], as pictures of "noncrossing partitions" of vertices around a circle. He noticed that the planarity of these pictures yields a natural antiautomorphism, which we call the Kreweras complement.

Definition 1.3. Given a noncrossing partition $w \in \mathrm{NC}(W, c) \subseteq W$, let $\operatorname{Krew}(w):=$ $c w^{-1}$. Since the reflection length $\ell_{T}$ is invariant under conjugation it follows that $\operatorname{Krew}(w)$ is also in $\mathrm{NC}(W, c)$.

In type $A_{n-1}$ the set $N C(W, c)$ is isomorphic to the lattice of classical noncrossing partitions: place the vertices $\{1,2, \ldots, n\}$ around a circle; we say a partition of these vertices is "noncrossing" if the convex hulls of its blocks are pairwise disjoint. To construct the classical Kreweras map, place the vertices $\left\{1,1^{\prime}, 2,2^{\prime}, \ldots, n, n^{\prime}\right\}$ around a circle; if $\pi$ is a noncrossing partition of the vertices $\{1, \ldots, n\}$, then $\operatorname{Krew}(\pi)$ is defined to be the coarsest partition of $\left\{1^{\prime}, 2^{\prime}, \ldots, n^{\prime}\right\}$ such that $\pi \cup$ $\operatorname{Krew}(\pi)$ is a noncrossing partition of $\left\{1,1^{\prime}, \ldots, n, n^{\prime}\right\}$. For example, Figure 2 shows a single orbit of Krew acting on the noncrossing partitions of a square (given by the black vertices). Note here that $\mathrm{Krew}^{2}$ rotates the square by $90^{\circ}$. 
For a general root system we have $\operatorname{Krew}^{2}(w)=c w c^{-1}$, which is just conjugation by the Coxeter element. Since any Coxeter element $c$ has order $h$ (indeed this is the usual definition of the Coxeter number $h$ ) we conclude that $\mathrm{Krew}^{2 h}$ is the identity map. Thus we will prove part (i) of the Panyushev Conjectures by constructing a bijection from antichains to noncrossing partitions that sends Pan to Krew.

1.3. Panyushev complement $=$ Kreweras complement. The main idea for our bijection is to essentially declare that $\mathrm{Pan}=\mathrm{Krew}$, and then work out what this means. The key observation is the following.

Since a Dynkin diagram of finite type is a tree, we can partition the simple reflections $S$ into sets $S=L \sqcup R$ such that the elements of $L$ commute pairwise, as do the elements of $R$. Let $c_{L}$ denote the product of the reflections $L$ (in any order) and similarly let $c_{R}$ denote the product of the reflections $R$. Thus $c_{L}$ and $c_{R}$ are involutions in $W$ and $c=c_{L} c_{R}$ is a special Coxeter element, called a/the bipartite Coxeter element.

The data for Pan consists of a choice of simple system $\Delta$ - which from now on we will partition as $\Delta=\Delta_{L} \sqcup \Delta_{R}$ - and the data for Krew consists of a Coxeter element - which from now on we will assume to be $c=c_{L} c_{R}$. With this in mind, Panyushev observed that his map has two distinguished orbits: one of size $h$ consisting of the sets of roots at each rank of the root poset; and one of size 2, namely $\left\{\Delta_{L}, \Delta_{R}\right\}$. Similarly, the Kreweras map on $\mathrm{NC}\left(W, c_{L} c_{R}\right)$ has two distinguished orbits: one of size $h$ consisting of

$$
c_{L}, c_{L} c_{R} c_{L}, \ldots, c_{R} c_{L} c_{R}, c_{R}
$$

and one of size 2 , namely $\{\mathbf{1}, c\}$. The attempt to match these orbits was the genesis of our bijection.

To understand its definition, we must first discuss parabolic recursion. Let $W_{J} \subseteq$ $W$ denote the parabolic subgroup generated by a subset $J \subseteq S$ of simple reflections and let $\Delta_{J} \subseteq \Phi_{J}^{+} \subseteq \Phi^{+}$be the corresponding simple and positive roots. Antichains and noncrossing partitions may be restricted to $W_{J}$ as follows. Given an antichain $I \subseteq \Phi^{+}$, its support $\operatorname{supp}(I)=\langle I\rangle \cap \Delta$ is the set of simple roots below $I$. If $\operatorname{supp}(I) \subseteq$ $J$, then $I$ is also an antichain in the parabolic subroot system $\Phi_{J}^{+}$. Similarly, the set $J$ induces a unique partition of the diagram $J=L_{J} \sqcup R_{J}$ with $L_{J} \subseteq L$ and $R_{J} \subseteq R$. Writing $c_{J}$ for $c_{L_{J}} c_{R_{J}}$, we may discuss the parabolic noncrossing partitions

$$
\mathrm{NC}\left(W_{J}, c_{J}\right) \subseteq \mathrm{NC}(W, c) .
$$

We fix these conventions for the statements of our Main Definition and Theorem.

Main Definition. Given an antichain $I \in \mathrm{NN}(W)$ we recursively define a noncrossing partition $\Theta_{W}(I) \in \mathrm{NC}(W, c)$ as follows. The initial condition is that $\Theta_{W}\left(\Delta_{L}\right):=1 \in W$.

(i) Choose $k \geq 0$ minimal so that $\operatorname{Pan}^{k}(I)$ has less than full support,

$$
\operatorname{supp}\left(\operatorname{Pan}^{k}(I)\right)=J \subsetneq S .
$$

(ii) Compute $w=\left(\prod_{s \in L \backslash J} s\right) \Theta_{W_{J}}(I) \in N C\left(W_{J}, c_{J}\right) \subseteq N C(W, c)$.

(iii) Finally, let $\Theta_{W}(I):=\mathrm{Krew}^{-k}(w) \in W$.

To show that this map is well defined, we have to show that every orbit contains at least one element which is not of full support (since otherwise it would sometimes be impossible to choose $k$ in step (i) above). 
Main Theorem. The map $\Theta_{W}$ is well defined and a bijection from nonnesting partitions to noncrossing partitions. Moreover, it is the unique map from $\mathrm{NN}(W)$ to $\mathrm{NC}(W, c)$ satisfying the following three properties:

(i) $\Theta_{W}\left(\Delta_{L}\right)=1 \in W$,

(initial condition)

(ii) $\Theta_{W} \circ \operatorname{Pan}=$ Krew $\circ \Theta_{W}$,

$(\operatorname{Pan}=\mathrm{Krew})$

(iii) $\Theta_{W}(I)=\left(\prod_{s \in L \backslash \operatorname{supp}(I)} s\right) \Theta_{W_{\text {supp }}(I)}(I)$.

(parabolic recursion)

Note that the Main Definition is uniform for root systems. It is also computationally efficient and has been implemented in Maple. In order to prove the Main Theorem, one has to ensure that properties (ii) and (iii) are compatible in the case of a Panyushev orbit containing multiple elements which are not of full support. One might hope for an explicit, nonrecursive definition of $\Theta_{W}$. We do provide such a definition in the classical types (though not uniformly). In contrast to the uniform character of the Main Definition, our proof of the Main Theorem is case-by-case, using the above-mentioned computation in the exceptional types.

We also note that the interaction between "nonnesting" and "noncrossing" properties is a subtle phenomenon, even just in type $A$ (see [9]). For the classical types in general: A. Fink and B. I. Giraldo [12 as well as M. Rubey and the second author 23] have both constructed NN to NC bijections that are uniform in a certain combinatorial framework that encompasses types $A, B / C$ and $D$. These bijections have some advantages over ours; in particular, both preserve the "parabolic type" of the nonnesting and noncrossing partitions. On the other hand, our new bijection has the advantages that 1) its definition is truly uniform, and 2) it allows us to prove the Panyushev Conjectures, as well as two conjectures of Bessis and Reiner, which we describe in the next section.

Since the original version of this paper appeared on the arxiv, Striker and Williams [26] found a new approach to the Panyushev complement, which allows them to construct equivariant bijections between noncrossing and nonnesting partitions in types $A$ and $B$ as special cases. They also give some additional references to occurrences of the Panyushev complement in the literature (prior to the work of Panyushev). Another recent paper related to the Panyushev complement is [24].

1.4. Cyclic sieving. The cyclic sieving phenomenon was introduced by V. Reiner, D. Stanton, and D. White in $[22$ as follows: let $X$ be a finite set, let $X(q) \in \mathbb{Z}[q]$ and let $\mathcal{C}_{d}=\langle c\rangle$ be a cyclic group of order $d$ acting on $X$. The triple $\left(X, X(q), \mathcal{C}_{d}\right)$ exhibits the cyclic sieving phenomenon (CSP) if

$$
[X(q)]_{q=\zeta^{k}}=\left|X^{c^{k}}\right|
$$

where $\zeta$ denotes a primitive $d$-th root of unity and $X^{c^{k}}:=\left\{x \in X: c^{k}(x)=x\right\}$ is the fixed-point set of $c^{k}$ in $X$. Let

$$
X(q) \equiv a_{0}+a_{1} q+\cdots+a_{d-1} q^{d-1} \bmod \left(q^{d}-1\right) .
$$

An equivalent way to define the CSP is to say that $a_{i}$ equals the number of $\mathcal{C}_{d^{-}}$orbits in $X$ whose stabilizer order divides $i$ [22, Proposition 2.1].

Bessis and Reiner recently showed that the action of the Coxeter element on noncrossing partitions together with a remarkable $q$-extension of the Catalan numbers 
Cat $(W)$ exhibits the CSP: define the $q$-Catalan number

$$
\operatorname{Cat}(W ; q):=\prod_{i=1}^{r} \frac{\left[d_{i}+h\right]_{q}}{\left[d_{i}\right]_{q}},
$$

where $[k]_{q}=1+q+q^{2}+\cdots+q^{k-1}$ is the usual $q$-integer. It is not obvious, but it turns out (see Berest, Etingof, and Ginzburg [4]) that this number is a polynomial in $q$ with nonnegative coefficients. In type $A_{n-1}$, the formula reduces to the classical $q$-Catalan number of Fürlinger and Hofbauer [13. That is, we have

$$
\operatorname{Cat}\left(A_{n-1} ; q\right)=\frac{1}{[n+1]_{q}}\left[\begin{array}{c}
2 n \\
n
\end{array}\right]_{q},
$$

where $\left[\begin{array}{l}a \\ b\end{array}\right]_{q}=\frac{[a]_{q} !}{[b]_{q} ![a-b]_{q} !}$ is the Gaussian binomial coefficient and $[k]_{q} !=[1]_{q}[2]_{q} \cdots[k]_{q}$ is the $q$-factorial.

For a Coxeter element $c \in W$, it follows directly from the definition that the map $\operatorname{conj}(w)=c w c^{-1}$ is a permutation of the set $\mathrm{NC}(W, c)$ of noncrossing partitions. In classical types, this corresponds to a "rotation" of the pictorial presentation.

Theorem 1.4 (Bessis and Reiner [6]). The triple $(\mathrm{NC}(W), \operatorname{Cat}(W ; q),\langle\operatorname{conj}\rangle)$ exhibits the CSP for any finite Coxeter group $W$.

Actually, they proved this result in the greater generality of finite complex reflection groups; we will restrict the current discussion to (crystallographic) finite real reflection groups - that is, finite Coxeter groups and finite Weyl groups, respectively. At the end of their paper, Bessis and Reiner [6] conjectured several other examples of cyclic sieving, two of which we will prove in this paper.

Theorem 1.5. Let $W$ be a finite Coxeter group, respectively finite Weyl group.

(i) The triple $(\mathrm{NC}(W), \operatorname{Cat}(W ; q),\langle\mathrm{Krew}\rangle)$ exhibits the CSP.

(ii) The triple $(\mathrm{NN}(W), \operatorname{Cat}(W ; q),\langle\mathrm{Pan}\rangle)$ exhibits the $C S P$.

Note that (i) is a generalization of Theorem 1.4 since $\mathrm{Krew}^{2}$ is the same as conjugation by the Coxeter element. The type $A$ version of (i) has been proved by D. White (see [6]) and independently by C. Heitsch [15] C. Krattenthaler has announced a proof of a more general version for complex reflection groups which appeared in the exceptional types in [18; and will appear for the group $G(r, p, n)$ in [17. In this paper we find it convenient to present an independent proof, on the way to proving our Main Theorem. Combining (i) and the Main Theorem then yields (ii) as a corollary.

1.5. Outline. The paper is organized as follows.

In Section 2, we introduce a notion of noncrossing handshake configurations for the classical types, and define a bijection $\phi_{W}$ from noncrossing handshake configurations $\mathcal{T}_{W}$ to noncrossing partitions $\mathrm{NC}(W, c)$. We establish the cyclic sieving phenomenon for noncrossing partitions using these bijections in classical types, and via a computer check for the exceptional types.

In Section 3, we define a bijection $\psi_{W}$ from the nonnesting partitions of $W$ to $\mathcal{T}_{W}$ in the classical types. Using this, we establish the cyclic sieving phenomenon for nonnesting partitions in the classical types, and again via a computer check for the exceptional types. 

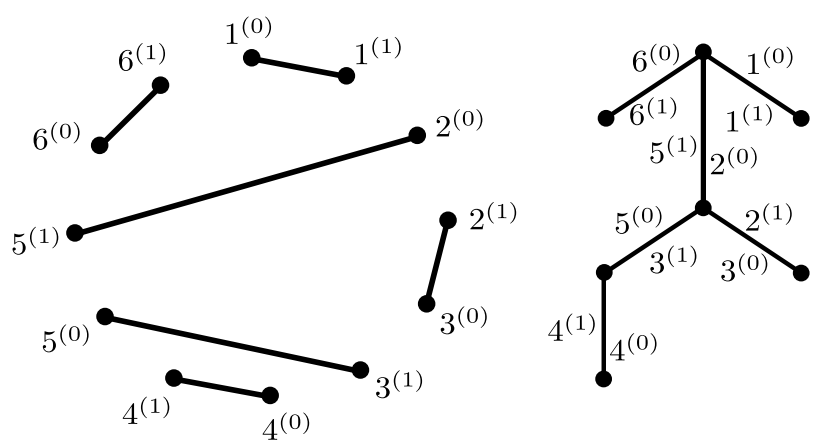

FiguRE 3. The noncrossing handshake configuration $T \in \mathcal{T}_{6}$ for $w=(2,3,5)$ and its associated rooted plane tree.

In Section 4, we show that the bijection from the nonnesting partitions of $W$ to $\mathcal{T}_{W}$ in the classical types satisfies a suitable notion of parabolic induction.

In Section 5, we put together the bijections from sections two and three to prove the Main Theorem. The calculations for the exceptional types were done using Maple code, which is available from the first author.

In the final section, Section 6, we use the combinatorics describing the Panyushev and the Kreweras complementation to prove the Panyushev Conjectures.

\section{The Kreweras CSP fOR NONCROSSing PARTitions}

In this section, we prove Theorem 1.5(i) for every type individually. For type $A_{n-1}$, C. Heitsch proved the theorem by connecting noncrossing partitions of type $A_{n-1}$ to noncrossing set partitions of $[n]:=\{1, \ldots, n\}$ and moreover to noncrossing handshake configurations of $[2 n]$ and to rooted plane trees. For the classical types, we will explore a connection which is related to the construction of C. Heitsch as described in Remark 2 .

2.1. Type $A$. Fix the linear Coxeter element $c$ to be the long cycle $(1,2, \ldots, n)$. Here, linear refers to the fact that it comes from a linear ordering of the Dynkin diagram. It is well known that the set of noncrossing partitions $\mathrm{NC}_{n}:=\mathrm{NC}\left(A_{n-1}\right)$ can be identified with the set of noncrossing handshake configurations. The ground set consists of 2 copies of $[n]$ colored by 0 and 1 drawn on a circle in the order $1^{(0)}, 1^{(1)}, \ldots, n^{(0)}, n^{(1)}$. A noncrossing handshake configuration is defined to be a noncrossing matching of those 2 copies of $[n]$; see Figure 3 . As shown in the figure, they are in natural bijection with rooted plane trees. The bijection $\phi_{A_{n-1}}: \mathcal{T}_{n} \longrightarrow$ $\mathrm{NC}_{n}$ is then, for $w=\phi_{A_{n-1}}(T)$, given by

$$
\left(i^{(1)}, j^{(0)}\right) \in T \Leftrightarrow w(i)=j .
$$

For a direct description of noncrossing partitions in terms of rooted plane trees see e.g. [5, Figure 6].

Remark 1. Observe that the described construction does not require the choice of the linear Coxeter element. As the Coxeter elements in type $A_{n-1}$ are exactly the long cycles, one obtains analogous constructions by labelling the vertices of $\mathcal{T}_{n}$ by any given long cycle. This corresponds to the natural isomorphism between 
$\mathrm{NC}(W, c)$ and $\mathrm{NC}\left(W, c^{\prime}\right)$ given by conjugation sending $c$ to the Coxeter element $c^{\prime}$. We will make use of this flexibility later on in this paper.

The following proposition follows immediately from the definition.

Proposition 2.1. The Kreweras complementation on $\mathrm{NC}_{n}$ can be described in terms of $\mathcal{T}_{n}$ by clockwise rotation of all edges by one, or, equivalently, by counterclockwise rotation of all vertex labels by one. I.e., for $T \in \mathcal{T}_{n}$, we have

$$
\left(i^{(1)}, j^{(0)}\right) \in T \Leftrightarrow\left(j^{(1)},(i+1)^{(0)}\right) \in \operatorname{Krew}(T) .
$$

Remark 2. One can easily deduce the proposition as well from O. Bernardi's description [5, Figure 6] and the definition of the Kreweras complementation of a set partition to be its coarsest complementary set partition. C. Heitsch obtains analogous results in [15] by directly considering a bijection $\phi^{\prime}$ between $\mathcal{T}_{n}$ and $\mathrm{NC}_{n}$ which is related to the bijection $\phi$ described above by $\phi^{\prime}(w)=\phi(\operatorname{Krew}(w))$.

For more readability, we set $\operatorname{Cat}_{n}(q):=\operatorname{Cat}\left(A_{n-1} ; q\right)$, and $\operatorname{Cat}_{n}:=\operatorname{Cat}_{n}(1)$.

Theorem 2.2. The triple $\left(\mathrm{NC}_{n}, \mathrm{Cat}_{n}(q),\langle\mathrm{Krew}\rangle\right)$ exhibits the CSP.

Proof. The theorem follows immediately from [16, Theorem 8]: let $d$ be an integer such that $d \mid 2 n$ and let $\zeta$ be a primitive $d$-th root of unity. Then it follows e.g. from [11. Lemma 3.2] that $\operatorname{Cat}_{n}(q)$ reduces for $q=\zeta$ to

$$
\left[\operatorname{Cat}_{n}(q)\right]_{q=\zeta}=\left\{\begin{array}{cl}
\mathrm{Cat}_{n} & \text { if } d=1, \\
n \mathrm{Cat}_{\frac{n-1}{2}} & \text { if } d=2 \text { and } n \text { odd } \\
\left(\begin{array}{c}
2 n / d \\
n / d
\end{array}\right) & \text { if } d \geq 2, d \mid n \\
0 & \text { otherwise. }
\end{array}\right.
$$

In [16, Theorem 8], C. Heitsch proved that noncrossing handshake configurations of $2 n$ which are invariant under a $d$-fold rotation, i.e., for which $\mathrm{Krew}^{2 n / d}(T)=T$, are counted by those numbers.

2.2. Types $B$ and $C$. As the reflection groups of types $B$ and $C$ coincide, the notions of noncrossing partitions do as well. Therefore we restrict our attention to type $C$. In this case, we fix the linear Coxeter element $c$ to be the long cycle $(1, \ldots, n,-1, \ldots,-n)$ and keep in mind that we could replace $c$ by any long cycle of analogous form. $\mathrm{NC}\left(C_{n}\right)$ can be seen as the subset of $\mathrm{NC}\left(A_{2 n-1}\right)$ containing all elements for which $i \mapsto j$ if and only if $-i \mapsto-j$, where $n+i$ and $-i$ are identified. $\mathcal{T}_{C_{n}}$ is defined to be the set of all noncrossing handshake configurations $T$ of $[ \pm n]$ for which $\left(i^{(1)}, j^{(0)}\right) \in T$ if and only if $\left(-i^{(1)},-j^{(0)}\right) \in T$. The Kreweras complementation on $\mathrm{NC}\left(C_{n}\right)$ is again the clockwise rotation of all edges by 1 . Observe that the symmetry property is expressed in terms of the Kreweras complementation by $\operatorname{Krew}^{2 n}(T)=T$ for $T \in \mathcal{T}_{C_{n}}$. By construction, the bijection $\phi_{A_{2 n-1}}: \mathcal{T}_{2 n} \stackrel{\sim}{\longrightarrow} \mathrm{NC}_{2 n}$ restricts to a bijection

$$
\phi_{C_{n}}: \mathcal{T}\left(C_{n}\right) \stackrel{\sim}{\longrightarrow} \mathrm{NC}\left(C_{n}\right),
$$

which is compatible with the Kreweras complementation, i.e.,

$$
\phi_{C_{n}}(\operatorname{Krew}(T))=\operatorname{Krew}\left(\phi_{C_{n}}(T)\right) .
$$

For the proof of Theorem 1.5(i) in type $C$, we need the following observation. 
Lemma 2.3. Let $d_{1}$ and $d_{2}$ be divisors of $2 n$, and let $d_{3}=\operatorname{lcm}\left\{d_{1}, d_{2}\right\} . T \in \mathcal{T}_{n}$ is invariant both under $d_{1}$ - and $d_{2}$-fold rotation if and only if $T$ is invariant under $d_{3}$-fold symmetry.

Proposition 2.4. The triple $\left(\mathrm{NC}\left(C_{n}\right), \operatorname{Cat}\left(C_{n} ; q\right),\langle\mathrm{Krew}\rangle\right)$ exhibits the CSP.

The proof in type $C$ is a simple corollary of the proof in type $A$.

Proof. The $q$-Catalan number $\operatorname{Cat}(W ; q)$ reduces for $W=C_{n}$ to

$$
\operatorname{Cat}\left(C_{n}, q\right)=\left[\begin{array}{c}
2 n \\
n
\end{array}\right]_{q} .
$$

Let $d$ be an integer such that $d \mid 4 n$ and let $\zeta$ be a primitive $d$-th root of unity. Then it follows again from [11, Lemma 3.2] that $\operatorname{Cat}\left(C_{n}, q\right)$ reduces for $q=\zeta$ to

$$
\left[\operatorname{Cat}\left(C_{n}, q\right)\right]_{q=\zeta}=\left\{\begin{array}{cl}
\left(\begin{array}{c}
4 n / d \\
2 n / d
\end{array}\right) & \text { if } d \text { even and } d \mid 2 n \\
\left(\begin{array}{c}
2 n / d \\
n / d
\end{array}\right) & \text { if } d \text { odd } \\
0 & \text { otherwise. }
\end{array}\right.
$$

Let $d \mid 4 n$. Then, by the previous lemma, the number of elements in $\mathcal{T}_{C_{n}}$ which are invariant under $d$-fold symmetry, i.e., for which $\mathrm{Krew}^{4 n / d}(T)=T$, are exactly those elements in $\mathcal{T}_{2 n}$ which are invariant under $\operatorname{lcm}\{d, 2\}$-fold symmetry. The proposition follows.

2.3. Type $D$. In this case, we fix the linear Coxeter element $c$ to be given by $(1, \ldots, n-1,-1, \ldots,-n+1)(n,-n)$. As in types $A$ and $C$, the noncrossing handshake configuration in type $D$ comes from noncrossing set partitions of type $D$ as defined in [3] by replacing every point $i$ by the two points $i^{(0)}$ and $i^{(1)}$, together with the appropriate restrictions, as described below.

Define a matching of

$$
\left\{ \pm 1^{(0)}, \pm 1^{(1)}, \ldots, \pm n^{(0)}, \pm n^{(1)}\right\}
$$

to be noncrossing of type $D_{n}$ if the points $\left\{ \pm 1^{(0)}, \pm 1^{(1)}, \ldots, \pm(n-1)^{(0)}, \pm(n-1)^{(1)}\right\}$ are arranged clockwise on a circle as in type $C_{n-1}$ and the points $\left\{ \pm n^{(0)}, \pm n^{(1)}\right\}$ form a small counterclockwise oriented square in the center of the circle, and the matching does not cross in this sense. A noncrossing handshake configuration $T$ of type $D_{n}$ is a noncrossing matching $T$ of type $D_{n}$, with the additional properties that $\left(i^{(1)}, j^{(0)}\right) \in T$ if and only if $\left(-i^{(1)},-j^{(0)}\right) \in T$ and that the size of

$$
M_{ \pm}:=\left\{\left(i^{(1)}, j^{(0)}\right) \in T: i \text { and } j \text { have opposite signs }\right\}
$$

is divisible by 4 . See Figure 4 for examples of noncrossing handshake configurations of type $D_{3}$.

As in the other types, we keep in mind that we could replace the linear Coxeter element by any Coxeter element to obtain labellings for the vertices of a noncrossing handshake configuration of type $D$.

Define the Kreweras complementation Krew on $D_{n}$ by rotating the labels of the outer circle counterclockwise and the labels of the inner circle clockwise; more 

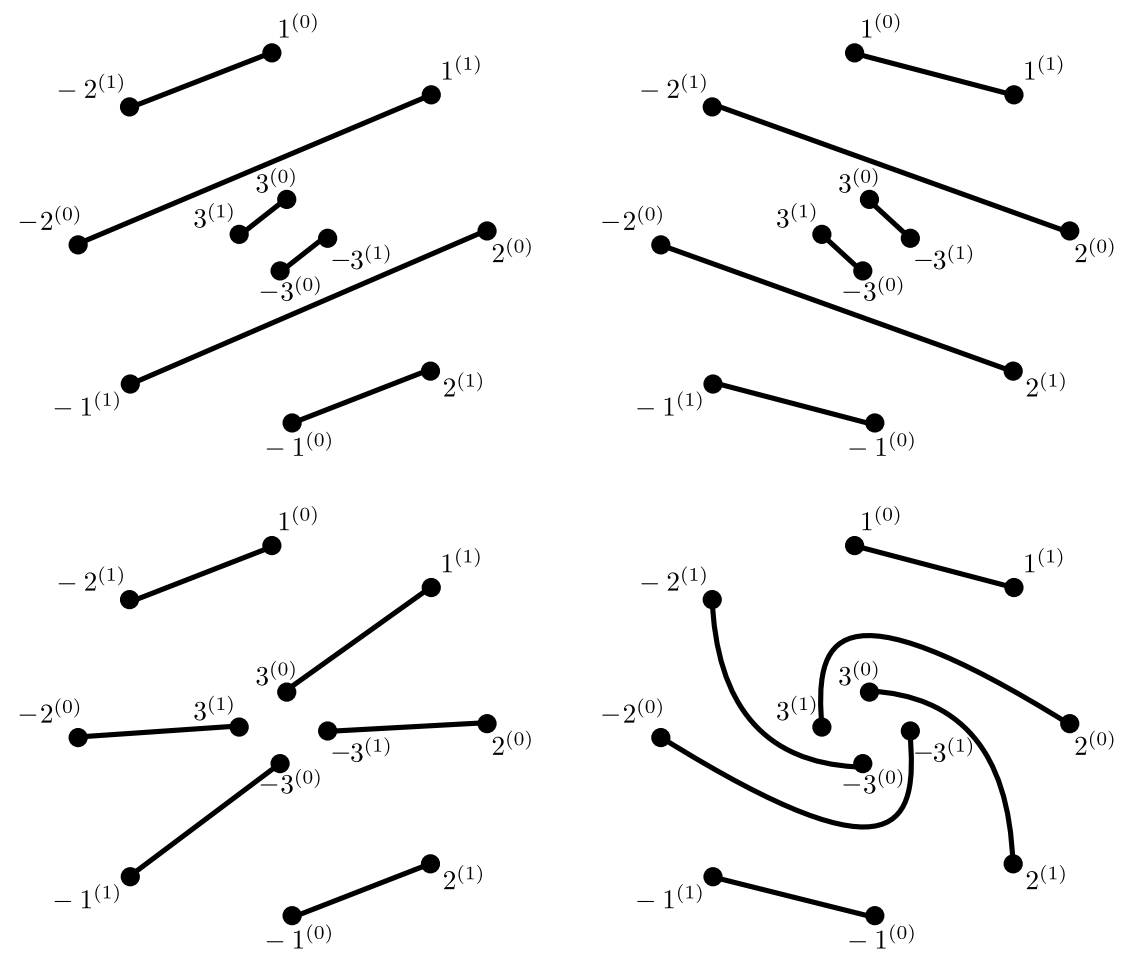

FiguRE 4. Four different noncrossing handshake configurations in $\mathcal{T}_{D_{3}}$.

precisely, let $\kappa\left(i^{(0)}\right):=i^{(1)}$ and

$$
\kappa\left(i^{(1)}\right):=\left\{\begin{array}{cl}
(i+1)^{(0)} & \text { if } i \in[n-2], \\
(i-1)^{(0)} & \text { if } i \in[-n+2], \\
(-1)^{(0)} & \text { if } i=n-1, \\
1^{(0)} & \text { if } i=-n+1, \\
(-n)^{(0)} & \text { if } i=n, \\
n^{(0)} & \text { if } i=-n .
\end{array}\right.
$$

Then $\left(i^{(1)}, j^{(0)}\right) \in T$ if and only if $\left(\kappa\left(j^{(0)}\right), \kappa\left(i^{(1)}\right)\right) \in \operatorname{Krew}(T)$. To see this, observe that the only outer vertices changing sign are $\pm(n-1)^{(1)}$, and the only two inner vertices are $\pm n^{(1)}$. Thus, the size of $M_{ \pm}$for $\operatorname{Krew}(T)$ is again divisible by 4 . As an immediate consequence of the construction in 3, we obtain that the map $\phi_{D_{n}}: \mathcal{T}_{D_{n}} \stackrel{\sim}{\longrightarrow} \mathrm{NC}\left(D_{n}\right)$ defined in the same way as for $\mathrm{NC}_{n}$ is well defined and a bijection between noncrossing handshake configurations of type $D_{n}$ and $\operatorname{NC}\left(D_{n}\right)$.

Proposition 2.5. The bijection $\phi_{D_{n}}: \mathcal{T}_{D_{n}} \stackrel{\sim}{\longrightarrow} \mathrm{NC}\left(D_{n}\right)$ is compatible with the Kreweras complementation, i.e., for $T \in \mathcal{T}_{D_{n}}$,

$$
\phi_{D_{n}}(\operatorname{Krew}(T))=\operatorname{Krew}\left(\phi_{D_{n}}(T)\right) .
$$

Proof. Let $\left(i^{(1)}, j^{(0)}\right) \in T$. This implies that $\left(\kappa\left(j^{(0)}, \kappa\left(i^{(1)}\right)\right)\right) \in \operatorname{Krew}(T)$. Therefore, by checking the different cases in (3), we obtain $\phi_{D_{n}}(\operatorname{Krew}(T)) \phi_{D_{n}}(T)=c$, and moreover, $\phi_{D_{n}}(\operatorname{Krew}(T))=c \phi_{D_{n}}(T)^{-1}=\operatorname{Krew}\left(\phi_{D_{n}}(T)\right)$.

Proposition 2.6. The triple $\left(\mathrm{NC}\left(D_{n}\right), \operatorname{Cat}\left(D_{n} ; q\right),\langle\mathrm{Krew}\rangle\right)$ exhibits the CSP. 


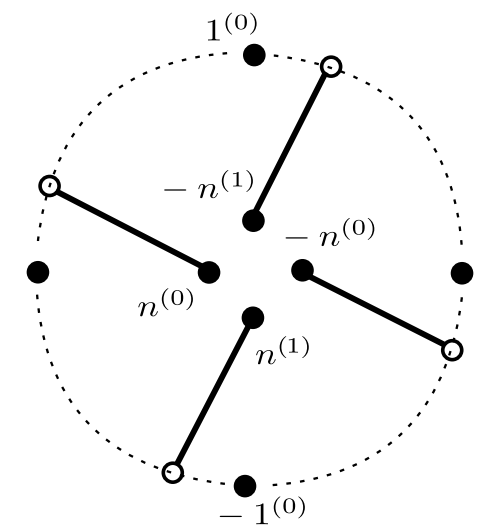

Figure 5. A typical situation in $\mathcal{T}_{D_{n}}$ with 4 -fold symmetry for $4=d \mid n$.

Proof. The $q$-Catalan number $\operatorname{Cat}\left(D_{n} ; q\right)$ is given by

$$
\operatorname{Cat}\left(D_{n}, q\right)=\left[\begin{array}{c}
2 n-1 \\
n
\end{array}\right]_{q^{2}}+q^{n}\left[\begin{array}{c}
2 n-2 \\
n
\end{array}\right]_{q^{2}} .
$$

Let $d$ be an integer such that $d \mid 4(n-1)$ and let $\zeta$ be a primitive $d$-th root of unity. Then it follows again from [11, Lemma 3.2] that $\operatorname{Cat}\left(D_{n}, q\right)$ reduces for $q=\zeta$ to

$$
\left[\operatorname{Cat}\left(D_{n}, q\right)\right]_{q=\zeta}=\left\{\begin{array}{cl}
\operatorname{Cat}\left(D_{n}\right) & \text { if } d=1, \\
\operatorname{Cat}\left(D_{n}\right) & \text { if } d=2, n \text { even, } \\
\operatorname{Cat}\left(C_{n-1}\right) & \text { if } d=2, n \text { odd, } \\
\operatorname{Cat}\left(C_{n / 2}\right) & \text { if } d=4,4 \mid n, \\
\operatorname{Cat}\left(C_{2(n-1) / d}\right) & \text { if } d \geq 4 \text { even, } d \mid 2(n-1), \\
\operatorname{Cat}\left(C_{(n-1) / d}\right) & \text { if } d \geq 3 \text { odd, } \\
0 & \text { otherwise. }
\end{array}\right.
$$

For $d=1$, this is obvious.

For $d=2, n$ even, the symmetry property implies that $\operatorname{Krew}^{2(n-1)}(T)=T$ for all $T \in \mathcal{T}_{D_{n}}$.

For $d=2, n$ odd, observe that $T \in \mathcal{T}_{D_{n}}$ is invariant under 2-fold symmetry, i.e., $\mathrm{Krew}^{2(n-1)}(T)=T$ if and only if $\left\{ \pm n^{(0)}, \pm n^{(1)}\right\}$ forms a submatching of $T$. Therefore, those are counted by $\operatorname{Cat}\left(C_{n-1}\right)$.

For $d=4 \mid n$, we want that $\mathrm{Krew}^{n-1}(T)=T$ and therefore, $\left\{ \pm n^{(0)}, \pm n^{(1)}\right\}$ must not form a submatching of $T$ and we are in a situation as indicated in Figure 5 , This gives

$$
\begin{aligned}
\left|\left\{T \in \mathcal{T}_{D_{n}}: \operatorname{Krew}^{n-1}(T)=T\right\}\right| & =2(n-1) \operatorname{Cat}\left(A_{(n-2) / 2}\right) \\
& =\frac{4(n-1)}{n}\left(\begin{array}{c}
n-2 \\
(n-2) / 2
\end{array}\right)=\left(\begin{array}{c}
n \\
n / 2
\end{array}\right),
\end{aligned}
$$

where the first 2 comes from the 2 -fold rotation of the inner square, the $n-1$ is the number of possible connections between the inner square and the circle, and 
$\operatorname{Cat}\left(A_{(n-2) / 2}\right)$ is the number of noncrossing handshake configurations of the $n-2$ free points on the outer circle.

For $d \geq 4$ even, $d \mid 2(n-1)$, we have again that $\left\{ \pm n^{(0)}, \pm n^{(1)}\right\}$ forms a submatching of $T$ and we have immediately that

$$
\left|\left\{T \in \mathcal{T}_{D_{n}}: \operatorname{Krew}^{4(n-1) / d}(T)=T\right\}\right|=\operatorname{Cat}\left(C_{2(n-1) / d}\right) .
$$

For $d \geq 3$ odd, it follows that $d \mid n-1$ and the same argument as in the previous case applies.

The only otherwise case which is left is the case $d \geq 4$ even, $d \nmid 2(n-1)$. In this case, we see that $4 \mid d$ and it follows together with the symmetry property that there does not exist a $T \in \mathcal{T}_{D_{n}}$ such that $\operatorname{Krew}^{4(n-1) / d}(T)=T$.

2.4. Type $I_{2}(k)$. For the dihedral groups, we obtain the theorem by straightforward computations. Let $I_{2}(k)=\langle a, b\rangle$ for two given simple reflections $a, b$ and fix the linear Coxeter element $c:=a b$. Then $\mathrm{NC}\left(I_{2}(k)\right)$ contains $\mathbf{1}, c$ and all $k$ reflections contained in $I_{2}(k)$.

Proposition 2.7. The triple $\left(\mathrm{NC}\left(I_{2}(k)\right)\right.$, Cat $\left.\left(I_{2}(k) ; q\right),\langle\mathrm{Krew}\rangle\right)$ exhibits the CSP.

Proof. The Kreweras complementation Krew on $\mathrm{NC}\left(I_{2}(k)\right)$ has 2 orbits, one is $\{\mathbf{1}, c\}$ and the other contains all $k$ reflections. On the other hand,

$$
\begin{aligned}
\operatorname{Cat}\left(I_{2}(k) ; q\right) & =\frac{[k+2]_{q}[2 k]_{q}}{[2]_{q}[k]_{q}} \\
& =\left\{\begin{array}{cl}
\left(1+q^{2}+\cdots+q^{k}\right)\left(1+q^{k}\right) & \text { if } k \text { even, } \\
1+q^{2}+\cdots+q^{k-1}+q^{k}+q^{k+1}+\cdots+q^{2 k} & \text { if } k \text { odd, }
\end{array}\right.
\end{aligned}
$$

and the proposition follows.

2.5. Exceptional types. For the exceptional Coxeter groups,

$$
\operatorname{Cat}(W ; q) \bmod \left(q^{2 h}-1\right)
$$

can be simply computed and by (11), we need to find the following orbit lengths, where $i * j$ is shorthand for $i$ orbits of length $j$ :

$$
\begin{aligned}
& F_{4}: 8 * 12,1 * 4,1 * 3,1 * 2, \\
& H_{3}: 3 * 10,1 * 2, \\
& H_{4}: 9 * 30,1 * 5,1 * 3,1 * 2, \\
& E_{6}: 30 * 24,8 * 12,1 * 8,1 * 4,1 * 3,1 * 2, \\
& E_{7}: 230 * 18,3 * 6,1 * 2, \\
& E_{8}: 832 * 30,5 * 15,3 * 10,2 * 5,1 * 3,1 * 2 .
\end{aligned}
$$

Those orbit lengths were verified with a computer; as mentioned above, they can be deduced as well from [18.

\section{The Panyushev CSP for nonnesting Partitions}

In this section, we prove Theorem 1.5(ii) for every type individually by providing a bijection between nonnesting partitions and noncrossing handshake configurations which maps the Panyushev complementation to the Kreweras complementation. We consider the same noncrossing handshake configurations as before, but we use a different labelling to refer to the vertices. In type $A_{n-1}$, we label the vertices 


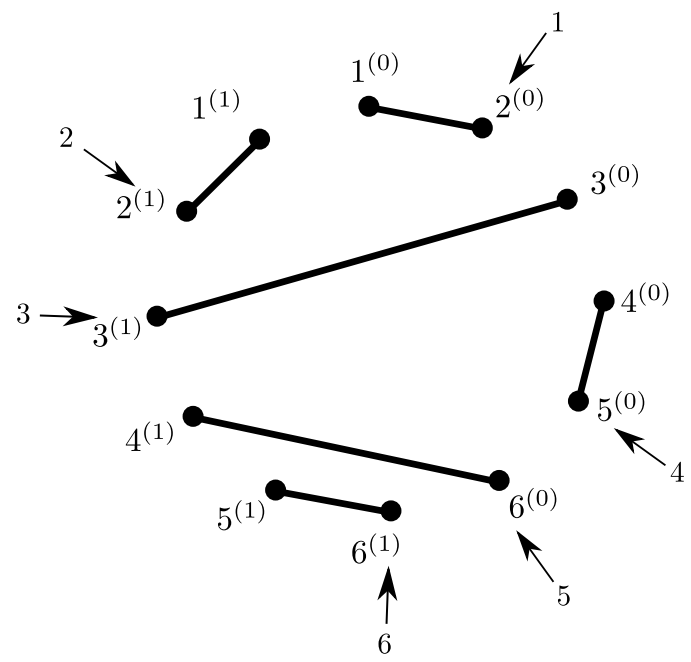

FiguRE 6 . The nonnesting labels on a noncrossing handshake configuration in $\mathcal{T}_{6}$.

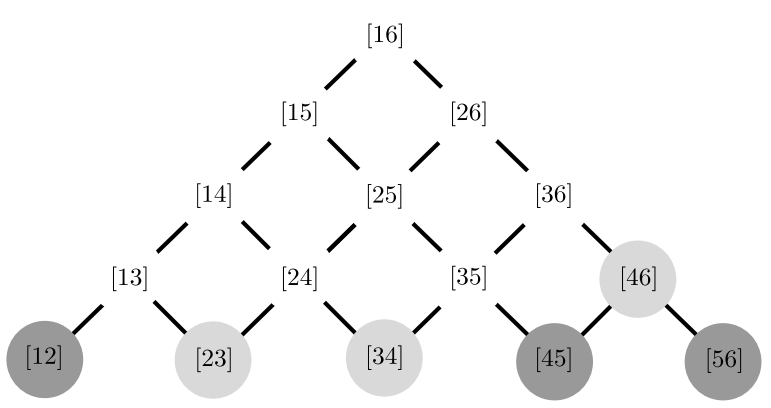

(a)

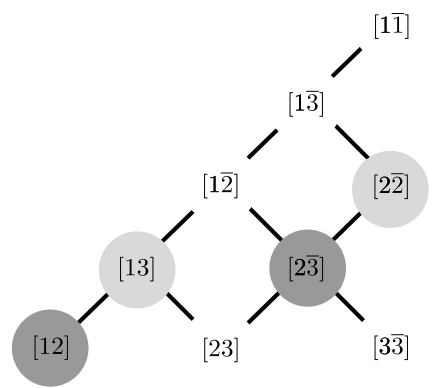

(b)

Figure 7. (a) An antichain and its image under the Panyushev complementation in the root poset of type $A_{5}$; (b) another antichain and its image in the root poset of type $C_{3}$.

on the outer circle by $\left\{1^{(0)}, \ldots, n^{(0)}, n^{(1)}, \ldots, 1^{(1)}\right\}$ in clockwise order. E.g., the noncrossing handshake configuration shown in Figure 3 is relabelled as shown in Figure 6.

3.1. Type $A$. Let $\Phi^{+}:=\left\{(i, j)=e_{i}-e_{j}: 1 \leq i<j \leq n\right\}$ be the set of all transpositions identified with a set of positive roots for $A_{n-1}$. The root poset structure on $\Phi^{+}$is given by

$$
(i, j) \leq\left(i^{\prime}, j^{\prime}\right) \Leftrightarrow i^{\prime} \leq i<j \leq j^{\prime}
$$

see Figure 77(a) for an example. Let $I=\left\{\left(i_{1}, j_{1}\right), \ldots,\left(i_{k}, j_{k}\right)\right\} \in \mathrm{NN}\left(A_{n-1}\right)$ such that $i_{1}<\cdots<i_{k}$. Observe that (44) implies $j_{1}<\cdots<j_{k}$ as well. Define a map

$$
\psi_{A_{n-1}}: \mathrm{NN}\left(A_{n-1}\right) \longrightarrow \mathcal{T}_{n}
$$


as follows: for $1 \leq \ell \leq k$, mark the vertex $j_{\ell}^{(0)}$ with $i_{\ell}$ and for $i \in[n] \backslash\left\{i_{1}, \ldots, i_{k}\right\}$ mark the vertex $i^{(1)}$ with $i$. Now, for $1 \leq i \leq n$, in increasing order, match the vertex marked with $i$ with the first nonmatched vertex, where first is interpreted counterclockwise from the marked vertex if $i \in\left\{i_{1}, \ldots, i_{k}\right\}$ and clockwise from the marked vertex if $i \notin\left\{i_{1}, \ldots, i_{k}\right\}$. For example, for the antichain

$$
I=\{(1,2),(4,5),(5,6)\} \in \mathrm{NN}\left(A_{5}\right)
$$

considered in Figure 7(a), we have $\psi_{A_{n-1}}(I)=T$, where $T \in \mathcal{T}_{6}$ is the noncrossing handshake configuration shown in Figures 3 and 6 .

To show that $\psi_{A_{n-1}}$ is a bijection, we define its inverse $\psi_{A_{n-1}}^{\prime}: \mathcal{T}_{n} \rightarrow \mathrm{NN}\left(A_{n-1}\right)$. Let $T \in \mathcal{T}_{n}$. Mark all $j^{(b)}$ for which $\left(i^{(a)}, j^{(b)}\right) \in T$ with $i<j$, or with $i=j$ and $(a, b)=(0,1)$. Next, label all marks $i^{(1)}$ with $i$, and then label all marks $i^{(0)}$ clockwise with the remaining labels in $[n]$. The antichain $\psi_{A_{n-1}}^{\prime}(T)$ is then given by

$$
\psi_{A_{n-1}}^{\prime}(T)=\left\{(i, j): \operatorname{vertex} j^{(0)} \text { is marked by } i\right\} .
$$

Proposition 3.1. The map $\psi_{A_{n-1}}^{\prime}$ is well defined and the inverse of $\psi_{A_{n-1}}$. In particular, $\psi_{A_{n-1}}: \mathrm{NN}\left(A_{n-1}\right) \stackrel{\sim}{\longrightarrow} \mathcal{T}_{n}$ is a bijection.

Proof. To see that $\psi_{A_{n-1}}^{\prime}$ is well defined, we have to check that any marked vertex $j^{(0)}$ is marked with some $i<j$. Assume that $j^{(0)}$ is marked with $j$. This implies that the set $\left\{1^{(0)}, \ldots,(j-1)^{(0)},(j-1)^{(1)}, \ldots, 1^{(1)}\right\}$ contains $j-1$ marked vertices and forms therefore a submatching, a contradiction to the fact that $j$, as it is marked, is matched to some element in this set.

As in the process of applying $\psi_{A_{n-1}}^{\prime}$ and of applying $\psi_{A_{n-1}}$ the same vertices get marked, $\psi_{A_{n-1}}^{\prime}$ is in fact the inverse of $\psi_{A_{n-1}}$.

Theorem 3.2. The bijection $\psi_{A_{n-1}}$ is compatible with the Panyushev respectively the Kreweras complementation. For $I \in \mathrm{NN}\left(A_{n-1}\right)$, we have

$$
\operatorname{Krew}\left(\psi_{A_{n-1}}(I)\right)=\psi_{A_{n-1}}(\operatorname{Pan}(I)) .
$$

To prove this theorem, we first have to understand how the Panyushev complementation behaves in type $A$. Recall that the $\operatorname{support} \operatorname{supp}(I)$ of some antichain $I \in \mathrm{NN}\left(A_{n-1}\right)$ is given by $\operatorname{supp}(I):=\bigcup_{(i, j) \in I}\left\{s_{i}, \ldots, s_{j-1}\right\}$. Next, set

$$
\hat{I}=\left\{\left(i_{1}^{\prime}, j_{1}^{\prime}\right), \ldots,\left(i_{k}^{\prime}, j_{k}^{\prime}\right)\right\}:=I \cup\left\{(i, i): s_{i-1}, s_{i} \notin \operatorname{supp}(I)\right\}
$$

such that $i_{1}^{\prime}<\cdots<i_{k}^{\prime}$, where the dummies $s_{0}, s_{n}$ are supposed not to be in $\operatorname{supp}(I)$. The Panyushev complementation is then given by

$$
\operatorname{Pan}(I)=\left\{\left(i_{2}^{\prime}-1, j_{1}^{\prime}+1\right), \ldots,\left(i_{k}^{\prime}-1, j_{k-1}^{\prime}+1\right)\right\} \in \mathrm{NN}\left(A_{n-1}\right) .
$$

Proposition 3.3. Let $I$ be a nonnesting partition. Then $s_{k} \notin \operatorname{supp}(I)$ if and only if $\left\{i^{(0)}, i^{(1)}: 1 \leq i \leq k\right\}$ defines a submatching of $\psi_{A_{n-1}}(I)$. In particular,

$$
\left(i^{(0)}, i^{(1)}\right) \in \psi_{A_{n-1}}(I) \Leftrightarrow(i, i) \in \hat{I} .
$$

Proof. The proposition follows directly from the definition.

Example 3.4. The noncrossing handshake configuration $T$ in Figure 6 is the image of $I=\{(1,2),(4,5),(5,6)\} \in \mathrm{NN}\left(A_{5}\right)$ under $\psi_{A_{5}}$. The complement of the support of $I$ is $S \backslash \operatorname{supp}(I)=\left\{s_{2}, s_{3}\right\}$. The submatchings guaranteed by the proposition are those on the vertices $\left\{1^{(0)}, 1^{(1)}, \ldots, k^{(0)}, k^{(1)}\right\}$ for $k \in\{2,3\}$. 
Proof of Theorem 3.2. As it is easier to see, we describe the analogous statement for $\psi_{A_{n-1}}^{\prime}$. The element $\psi_{A_{n-1}}^{\prime}(\operatorname{Krew}(T))$ can be described in terms of $\psi_{A_{n-1}}^{\prime}(T)$ as follows: a marked $i^{(0)}$ is turned to a marked $(i+1)^{(0)}$ (unless $i=n$ when the mark disappears), and for a marked $i^{(1)}$, we obtain a marked $(i-1)^{(1)}$ (unless $i=1$ when the mark disappears). If $\left(i^{(0)}, i^{(1)}\right) \in T$, the marked $i^{(1)}$ is replaced by a marked $(i+1)^{(0)}$. The theorem follows with Proposition 3.3 and the description of $\operatorname{Pan}(I)$ in terms of $\hat{I}$.

3.2. Types $B$ and $C$. In contrast to the situation for reflection groups, the notion of the root system does not coincide for types $B$ and $C$. The resulting root posets turn out to be isomorphic (as posets) but not equal. Thus, it suffices to study the Panyushev complementation on one of the two. As the connection between the root poset of type $C_{n}$ and the root poset of type $A_{2 n-1}$ is straightforward, whereas there is a little more work to do in type $B_{n}$, we will study nonnesting partitions of type $C_{n}$. This corresponds to the fact that the type $C_{n}$ Dynkin diagram can be obtained from the type $A_{n-1}$ Dynkin diagram through a "folding process".

The set of reflections identified with a set of positive roots in type $C_{n}$ is given by

$$
\Phi^{+}:=\left\{(i, j)=e_{i}-e_{j}: 1 \leq i<j \leq n\right\} \cup\left\{(i, \bar{j})=e_{i}+e_{j}: 1 \leq i \leq j \leq n\right\} .
$$

See Figure 7 (b) for the root poset of type $C_{3}$ as an example.

To understand nonnesting partitions of type $C_{n}$, observe that an antichain in $\Phi^{+}$can be identified with a symmetric antichain in the root poset of type $A_{2 n-1}$ : there is an involution $\delta$ on $\mathrm{NN}\left(A_{n-1}\right)$ by horizontally flipping the root poset of type $A_{n-1}$, i.e., replacing the positive root $(i, j)$ by $(n+1-j, n+1-i)$. In other words, $\delta$ is the induced map coming from the involution on the Dynkin diagram sending one linear ordering to the other. Define an antichain $I \in \mathrm{NN}\left(A_{n-1}\right)$ to be symmetric if it is invariant under this involution. It is well known that $\mathrm{NN}\left(C_{n}\right)$ can be seen as the set of all antichains $A \in \mathrm{NN}\left(A_{2 n-1}\right)$ which are symmetric,

$$
\mathrm{NN}\left(C_{n}\right) \cong\left\{I \in \mathrm{NN}\left(A_{2 n-1}\right): \delta(I)=I\right\} .
$$

Moreover, this identification is compatible with the Panyushev complementation,

$$
\delta(I)=I \Leftrightarrow \delta(\operatorname{Pan}(I))=\operatorname{Pan}(I) .
$$

This allows us to study this complementation on nonnesting partitions of type $C_{n}$ in terms of symmetric nonnesting partitions of type $A_{2 n-1}$.

On the other hand, we have seen above that the bijection $\phi_{A_{2 n-1}}: \mathcal{T}_{2 n} \longrightarrow$ $\mathrm{NC}\left(A_{2 n-1}\right)$ restricts to a bijection $\phi_{C_{n}}: \mathcal{T}_{C_{n}} \longrightarrow \mathrm{NC}\left(C_{n}\right)$. Therefore, we want to show that the bijection $\psi_{A_{2 n-1}}: \mathrm{NN}\left(A_{2 n-1}\right) \longrightarrow \mathcal{T}_{2 n}$ gives rise to a bijection $\psi_{C_{n}}: \mathrm{NN}\left(C_{n}\right) \longrightarrow \mathcal{T}_{C_{n}}$ which is again compatible with the Panyushev and the Kreweras complementations.

Lemma 3.5. The involution $\delta$ on $I$ for $I \in \mathrm{NN}\left(A_{n-1}\right)$ can be described in terms of the Kreweras complementation as

$$
\psi_{A_{n-1}}(\delta(I))=\operatorname{Krew}^{n}\left(\psi_{A_{n-1}}(I)\right) .
$$

Proof. For $T \in \mathcal{T}_{n}$, we have

$$
\left(i^{(a)}, j^{(b)}\right) \in T \Leftrightarrow\left((n+1-j)^{\left(b^{c}\right)},(n+1-i)^{\left(a^{c}\right)}\right) \in \operatorname{Krew}^{n}(T),
$$


where $a, b \in\{0,1\}$ and $a^{c}$ (resp. $b^{c}$ ) denotes the complement of $a$ (resp. $b$ ) in $\{0,1\}$. It is straightforward to check that this observation implies that

$$
\psi_{A_{n-1}}^{\prime}\left(\operatorname{Krew}^{n}\left(\psi_{A_{n-1}}(I)\right)\right)=\delta(I) .
$$

Theorem 3.6. $\psi_{A_{2 n-1}}$ restricts to a well-defined bijection $\psi_{C_{n}}: \mathrm{NN}\left(C_{n}\right) \longrightarrow \mathcal{T}_{C_{n}}$.

Proof. The statement of the theorem is equivalent to the statement that

$$
\delta(I)=I \Leftrightarrow \operatorname{Krew}^{n}\left(\psi_{A_{2 n-1}}(I)\right)=\psi_{A_{2 n-1}}(I) .
$$

This follows directly from the previous lemma.

3.3. Type $D$. Fix the numbering of the Dynkin diagram of type $D_{n}$ so that $n-2$ is adjacent to $n-1, n$, and $n-3$. We consider the involution $\delta$ of this diagram which interchanges $n$ and $n-1$. It acts on $\mathrm{NN}\left(D_{n}\right), \mathrm{NC}\left(D_{n}\right)$, and $\mathcal{T}_{D_{n}}$. On $\mathcal{T}_{D_{n}}$, it acts by rotating the inner four vertices by a half turn. It is convenient to define a new type of noncrossing handshake configuration, which we denote $\mathcal{T}_{D_{n} / \delta}$ : this consists of $4 n-4$ external vertices, labelled as in a $C_{n-1}$ noncrossing handshake configuration, such that either all the vertices participate in a $180^{\circ}$-rotationally symmetric noncrossing matching (in which case we simply have a $C_{n-1}$ noncrossing handshake configuration) or else all but four vertices participate in a $180^{\circ}$-rotationally symmetric noncrossing matching, while the four remaining vertices are isolated but have the property that any two of them could be attached without creating any crossings. It is clear that elements of $\mathcal{T}_{D_{n} / \delta}$ correspond to $\delta$-orbits in $\mathcal{T}_{D_{n}}$.

3.3.1. Defining a map from $\mathrm{NN}\left(D_{n}\right) / \delta$ to $\mathcal{T}_{D_{n} / \delta}$. Note that Krew acts naturally on $\mathcal{T}_{D_{n} / \delta}$, while Pan acts naturally on $\delta$-orbits in $\mathrm{NN}\left(D_{n}\right)$. We will begin by showing that $\left(\mathcal{T}_{D_{n} / \delta}\right.$, Krew) and $\left(\mathrm{NN}\left(D_{n}\right) / \delta\right.$, Pan $)$ are isomorphic as sets with a cyclic action.

In this subsection, we will define a cardinality-preserving bijection from $\delta$-orbits in $\mathrm{NN}\left(D_{n}\right)$ to $\mathcal{T}_{D_{n} / \delta}$, which we will denote by $\psi_{D_{n} / \delta}$. (In fact, for notational convenience, we will write $\psi_{D_{n} / \delta}$ as a map from $\mathrm{NN}\left(D_{n}\right)$ to $\mathcal{T}_{D_{n} / \delta}$ which is constant on $\delta$-orbits.) We will then show that it is possible to refine $\psi_{D_{n} / \delta}$ to a bijection from $\mathrm{NN}\left(D_{n}\right)$ to $\mathcal{T}_{D_{n}}$.

Singleton $\delta$-orbits in $\mathcal{T}_{D_{n} / \delta}$. Such an element consists of a type $C_{n-1}$ noncrossing handshake configuration on $4 n-4$ external vertices $1^{(0)}, \ldots,(2 n-2)^{(0)},(2 n-$ $2)^{(1)}, \ldots 1^{(1)}$.

Singleton $\delta$-orbits in $\mathrm{NN}\left(D_{n}\right)$. Such an element of $\mathrm{NN}\left(D_{n}\right)$ corresponds to a single element of $\mathrm{NN}\left(B_{n-1}\right)$. We reinterpret this as an element of $\mathrm{NN}\left(C_{n-1}\right)$, which corresponds (as we have already seen) to an element of $\mathrm{NN}\left(A_{2 n-3}\right)$ fixed under the involution of the $A_{2 n-3}$ diagram.

Map from singleton $\delta$-orbits in $\mathrm{NN}\left(D_{n}\right)$ to $\mathcal{T}_{D_{n}}$. We define $\psi_{D_{n} / \delta}$ on a singleton $\delta$-orbit by sending the type $A_{2 n-3}$ antichain to an $A_{2 n-3}$ noncrossing handshake configuration, using $\psi_{A_{2 n-3}}$.

Now we consider the doubleton $\delta$-orbits. Write $H$ for the $2 n-2$ vertices $\{(n-$ $\left.2)^{(1)}, \ldots, 1^{(1)}, 1^{(0)}, \ldots, n^{(0)}\right\}$, and $H^{c}$ for the other $2 n-2$ vertices on the boundary.

Doubleton $\delta$-orbits in $\mathcal{T}_{D_{n}}$. These correspond to elements of $\mathcal{T}_{D_{n} / \delta}$ which have four vertices of degree zero.

Doubleton $\delta$-orbits in $\mathrm{NN}\left(D_{n}\right)$. Let $I$ be an antichain in such an orbit. Write $\bar{I}$ for the collection of type $A_{2 n-3}$ roots obtained by taking each root in $I$, passing first 
to $B_{n-1}$, identifying the root poset of $B_{n-1}$ with that of $C_{n-1}$, and then unfolding to one or two roots in $A_{2 n-3}$. Note that $\bar{I}$ is typically not an antichain.

Example 3.7. Consider the $D_{n}$ antichain consisting of $\alpha_{n}+\alpha_{n-2}$ and $\alpha_{n-1}$. The former contributes elements $(n-1, n+1)$ and $(n-2, n)$, while the latter contributes $(n-1, n)$. This does not form an antichain. There will often be two elements in $\bar{I}$ with first coordinate $n-1$, and two elements with second coordinate $n$.

We also associate to $I$ an antichain in $\Phi_{A_{2 n-3}}$, defined as follows. Consider the elements of $\bar{I}$ which lie in the square with opposite corners at $(1,2 n-2)$ and $(n-1, n)$. (We call this square $R$.) Record the first coordinates of these as $i_{1}, \ldots, i_{r}$, and the last as $j_{1}, \ldots, j_{r}$.

Note that $j_{1}=j_{2}$ and $i_{r}=i_{r-1}$ are possible (occurring when $\bar{I}$ is not an antichain). Define $\widehat{I}$ by replacing these $r$ elements of $\bar{I}$ by the $r-1$ elements $\left(i_{1}, j_{2}\right),\left(i_{2}, j_{3}\right), \ldots,\left(i_{r-1}, j_{r}\right)$. (In the case that $r=1$, the result is that $\widehat{I} \cap R=\emptyset$.)

The map from doubleton $\delta$-orbits in $\mathrm{NN}\left(D_{n}\right)$ to doubleton $\delta$-orbits in $\mathrm{NC}\left(D_{n}\right)$. We define $\psi_{D_{n} / \delta}(I)$ in several steps. Using Lemma 3.9. below, we know that $\widehat{I} \in$ $\mathrm{NN}\left(C_{n-1}\right)$. Therefore, we can consider $\psi_{C_{n-1}}(\widehat{I}) \in \mathcal{T}_{C_{n-1}}$. Lemma 3.11 below guarantees that there are at least two edges in this diagram which run from vertices in $H$ to vertices in $H^{c}$. Remove the two such edges which are closest to the center. The result is a noncrossing handshake configuration of type $D_{n} / \delta$ as defined above. This is $\psi_{D_{n} / \delta}(I)$.

3.3.2. Defining $\psi_{D_{n}}$. We now consider refining $\psi_{D_{n} / \delta}$ to a map from $\mathrm{NN}\left(D_{n}\right)$ to $\mathcal{T}_{D_{n}}$.

We use the convention that a type $D$ noncrossing handshake configuration has the same outside labels as for type $D / \delta$ noncrossing handshake configurations, with four internal vertices which are numbered by congruence classes modulo 4 , increasing in counterclockwise order. We count as "positive", external vertices with label (0), and the internal vertices 0 and 3 , and as "negative", external vertices with the label (1) and the internal vertices 1 and 2 . In a noncrossing handshake configuration, the number of edges that connect a positive vertex to a negative vertex must be divisible by 4 .

If a noncrossing handshake configuration $T$ of type $D_{n} / \delta$ has no isolated vertices, this requirement means that there is a unique way of completing $T$ to a type $D_{n}$ configuration, while if $T$ has four isolated vertices, then there are two ways of completing $T$ to a type $D_{n}$ configuration.

For $a, b$ outer vertices, write $d(a, b)$ for the clockwise distance from $a$ to $b$. Write $e_{I}(a, b)$ for the number of vertices in the clockwise interval from $a$ to $b$, including $b$ but not $a$, and which are not on the clockwise end of an edge in $\psi_{D_{n} / \delta}(I)$.

For $I$ an antichain in $\mathrm{NN}\left(D_{n}\right)$ in a doubleton $\delta$-orbit, define $s(I)$ to be 0 if the root of $I$ whose image in $\bar{I}$ is $(i, n)$ with $i$ as small as possible has $\alpha_{n-1}$ in its support; otherwise, set $s(I)=1$.

We now define $\psi_{D_{n}}(I)$. If $I$ is in a singleton $\delta$-orbit, then define $\psi_{D_{n}}(I)$ to be $\psi_{D_{n} / \delta}(I)$ together with edges connecting the internal vertices in the unique possible way.

If $I$ is in a doubleton $\delta$-orbit, define $\psi_{D_{n}}(I)$ by starting with $\psi_{D_{n} / \delta}(I)$ and, for each singleton external vertex $v$, attach it to the internal vertex whose number is given by $n-d\left(v,(n-1)^{(0)}\right)+2 s(I)+2 e_{I}\left(v,(n-1)^{(0)}\right)$. 
Example 3.8. For the root poset of type $D_{3}$ with simple roots

$$
\alpha_{1}=e_{1}-e_{2}, \alpha_{2}=e_{2}-e_{3}, \alpha_{3}=e_{2}+e_{3},
$$

the four antichains $\emptyset,\left\{\alpha_{1}, \alpha_{2}, \alpha_{3}\right\},\left\{\alpha_{2}\right\},\left\{\alpha_{1}, \alpha_{3}\right\}$ are mapped by $\psi_{D_{n}}$ to the four noncrossing handshake configurations in $\mathcal{T}_{D_{3}}$ shown in Figure 4 from left to right.

3.3.3. Proof that $\psi_{D_{n}}$ is well defined and is a bijection. There are several lemmas which must be established to show that the definition given above makes sense, and yields a bijection.

Lemma 3.9. $\widehat{I}$ is in $\mathrm{NN}\left(A_{2 n-3}\right)$. Further, the map from $I$ to $\widehat{I}$ is injective, and its image consists of all the antichains in $\mathrm{NN}\left(C_{n-1}\right)$ (thought of as a subset of $\left.\mathrm{NN}\left(A_{2 n-3}\right)\right)$ except those containing $(n-1, n)$.

Proof. The inverse map is clear, since $i_{r}$ must be $n$ and $j_{1}$ must be $n-1$. This inverse map can be applied to any antichain in $\mathrm{NN}\left(C_{n-1}\right)$ except those containing $(n-1, n)$.

Now, since $\widehat{I}$ is in $\mathrm{NN}\left(C_{n-1}\right)$, its image under the bijection $\psi_{A_{2 n-3}}$ is a type $C_{n-1}$ noncrossing handshake configuration. The following lemma is useful.

Lemma 3.10. The image of $\psi_{C_{n-1}}$ applied to antichains with no roots in $R$ consists exactly of those type $C_{n-1}$ noncrossing handshake configurations with no edges from $\left\{(n-1)^{(1)}, \ldots, 1^{(1)}, 1^{(0)}, \ldots,(n-1)^{(0)}\right\}$ to the other vertices.

Proof. The first $n-1$ edges in the noncrossing handshake configuration will all connect vertices in $\left\{(n-1)^{(1)}, \ldots, 1^{(1)}, 1^{(0)}, \ldots,(n-1)^{(0)}\right\}$, which uses up all those vertices.

Lemma 3.11. The image of $\psi_{C_{n-1}}$ applied to $\widehat{I}$ for $I \in \mathrm{NN}\left(D_{n}\right)$ consists of exactly those type $C_{n-1}$ noncrossing handshake configurations with the property that there is at least one edge (and therefore at least two edges) from $H$ to $H^{c}$.

Proof. We have already shown that as $I$ runs through $\mathrm{NN}\left(D_{n}\right)$, we have that $\widehat{I}$ runs through those antichains in $\mathrm{NN}\left(C_{n-1}\right)$ not containing $(n-1, n)$. The image under $\mathrm{Pan}^{-1}$ of type $C_{n-1}$ antichains not containing $(n-1, n)$ is exactly the $C_{n-1}$ antichains whose intersection with $R$ is nonempty. Now apply Lemma 3.10 to $\operatorname{Pan}^{-1}(\widehat{I})$, together with the fact that Krew $\circ \psi_{C_{n-1}}=\psi_{C_{n-1}} \circ$ Pan.

We now have the pieces in place to establish the following proposition:

Proposition 3.12. The map $\psi_{D_{n} / \delta}$ is a bijection from $\mathrm{NN}\left(D_{n} / \delta\right)$ to $\mathcal{T}_{D_{n} / \delta}$.

Proof. It is clear that $\psi_{D_{n} / \delta}$ takes singleton $\delta$ orbits in $\mathrm{NN}\left(D_{n}\right)$ bijectively to the noncrossing handshake configurations in $\mathcal{T}_{D_{n} / \delta}$ which contain no isolated vertices. It is also clear that $\psi_{D_{n} / \delta}$ is an injection from doubleton orbits in $\mathrm{NN}\left(D_{n}\right)$ into the $\mathcal{T}_{D_{n} / \delta}$ noncrossing handshake configurations with four isolated vertices. Finally, given such a diagram, there is a unique way to reattach the isolated vertices to obtain a $\mathcal{T}_{C_{n-1}}$ noncrossing handshake configuration such that the reattached edges cross from $H$ to $H^{c}$. It follows that $\psi_{D_{n} / \delta}$ is a bijection.

We now proceed to show that $\psi_{D_{n}}$, as defined above, is a bijection from $\mathrm{NN}\left(D_{n}\right)$ to $\mathcal{T}_{D_{n}}$. To begin with, we need the following lemma which gives a condition equivalent to the parity condition on the number of edges in a type $D_{n}$ noncrossing handshake configuration which connect positive and negative vertices. 
Lemma 3.13. The condition that the number of edges joining a positive vertex to a negative vertex be divisible by four is equivalent to the condition that a positive, evennumbered singleton vertex must be connected to an internal vertex of odd parity, and similarly for the other possible choices of singleton vertex, where changing either "positive" or "even-numbered" reverses the parity of the internal vertex.

We are now ready to prove that $\psi_{D_{n}}$ is a bijection.

Lemma 3.14. $\psi_{D_{n}}$ is a bijection from $\mathrm{NN}\left(D_{n}\right)$ to $\mathcal{T}_{D_{n}}$.

Proof. We must show that if $v$ and $v^{\prime}$ are singleton vertices in $\psi_{D_{n} / \delta}(I)$, such that the next singleton vertex after $v$ in counterclockwise order is $v^{\prime}$, then the vertex to which $v^{\prime}$ is attached is one step counterclockwise from that to which $v$ is attached. We evaluate $-d\left(v^{\prime},(n-1)^{(0)}\right)+d\left(v,(n-1)^{(0)}\right)=-d\left(v^{\prime}, v\right)$ by counting the vertices between $v^{\prime}$ and $v$ (including $v$ but not $v^{\prime}$ ). Each edge on the outer rim between $v$ and $v^{\prime}$ contributes -2 to $-d\left(v^{\prime}, v\right)$ (one for each of its endpoints), and also contributes 2 to $2 e_{I}\left(v^{\prime},(n-1)^{(0)}\right)-2 e_{I}\left(v,(n-1)^{(0)}\right)=2 e_{I}\left(v^{\prime}, v\right)$. The only other contribution to $2 e_{I}\left(v^{\prime}, v\right)$ is an additional 2 coming from the vertex $v$, and also $-d\left(v^{\prime}, v\right)$ has an additional -1 coming from $v$. Thus the total effect is that $v^{\prime}$ is attached one step counterclockwise from $i$.

The condition provided by Lemma 3.13 is also clear from the definition. (Note that the complicated terms don't have any effect on the parity of the vertex to which we connect $v$.)

Bijectivity follows from bijectivity for $\psi_{D_{n} / \delta}$ together with the fact that the two elements of a doubleton $\delta$ orbit in $\mathrm{NN}\left(D_{n}\right)$ will be mapped to different noncrossing handshake configurations.

3.3.4. Compatibility between Panyushev complementation and rotation. We will first prove that $\psi_{D_{n} / \delta}$ expresses the compatibility between Panyushev complementation for $\mathrm{NN}\left(D_{n}\right) / \delta$ and rotation of $D_{n} / \delta$ noncrossing handshake configurations, and then we will prove the similar result for $\psi_{D_{n}}$.

Proposition 3.15. For $I \in \mathrm{NN}\left(D_{n}\right)$, we have that

$$
\psi_{D_{n} / \delta}(\operatorname{Pan}(I))=\operatorname{Krew}\left(\psi_{D_{n} / \delta}(I)\right) .
$$

Proof. We consider three cases separately. The first case is the case that $I$ is in a singleton $\delta$-orbit, in which case the result follows immediately from the analogous result for type $C_{n-1}$.

The second case is when $\widehat{I} \cap R \neq \emptyset$.

Lemma 3.16. If $\widehat{I} \cap R \neq \emptyset$, then $\widehat{\operatorname{Pan}(I)}=\operatorname{Pan}(\widehat{I})$ and

$$
\psi_{C_{n-1}}(\operatorname{Pan}(\widehat{I}))=\operatorname{Krew}\left(\psi_{C_{n-1}}(\widehat{I})\right)=\operatorname{Krew}\left(\psi_{D_{n} / \delta}(I)\right) .
$$

Proof. The fact that $\widehat{\operatorname{Pan}(I)}=\operatorname{Pan}(\widehat{I})$ in this case follows from the definitions. The compatibility of Pan and Krew in type $C$ implies that

$$
\psi_{C_{n-1}}(\operatorname{Pan}(\widehat{I}))=\operatorname{Krew}\left(\psi_{C_{n-1}}(\widehat{I})\right) .
$$

Finally, we wish to show that $\operatorname{Krew}\left(\psi_{C_{n-1}}(\widehat{I})\right)=\operatorname{Krew}\left(\psi_{D_{n} / \delta}(I)\right)$. The result which has to be established is that the pair of innermost edges in $\operatorname{Krew}\left(\psi_{C_{n-1}}(\widehat{I})\right)$ is the rotation of the innermost edges of $\psi_{C_{n-1}}(\widehat{I})$. This is true because, in order for the innermost edges no longer to be innermost, they must no longer run between 
the two sides of the diagram. But this would then imply that there were no edges between $H$ and $H^{c}$ in $\psi_{C_{n-1}}(\widehat{\operatorname{Pan}(I)})$, contrary to Lemma 3.11

We now consider the case that $\widehat{I} \cap R=\emptyset$. In this case, in contrast to the previous one, the proof does not pass through the similar statement in type $C$.

Let $\widehat{X}=\operatorname{Pan}(\widehat{I})$. It is immediate from the definition of Panyushev complementation that $\widehat{X} \cap R=(n-1, n)$. By Lemma 3.11, it follows that $\psi_{C_{n-1}}(\widehat{X})$ has no edges from $H$ to $H^{c}$.

By the compatibility of Pan and Krew in type $C$, we have that $\psi_{C_{n-1}}(\widehat{X})=$ $\operatorname{Krew}\left(\psi_{C_{n-1}}(\widehat{I})\right)$. The innermost edges of $\psi_{C_{n-1}}(\widehat{I})$ connecting $H$ to $H^{c}$, after rotation, no longer connect $H$ to $H^{c}$. Thus, in $\psi_{C_{n-1}}(\widehat{X})$, those edges connect $(n+1)^{(0)}$ to some $z^{\prime}$ in $H^{c}$ and $(n-2)^{(1)}$ to some (symmetrical) $z$ in $H$.

Lemma 3.17. $\psi_{C_{n-1}}(\widehat{\operatorname{Pan}(I)})$ can be obtained from $\psi_{C_{n-1}}(\widehat{X})$ by removing the edges connected to $(n+1)^{(0)}$ and $(n-2)^{(1)}$ and replacing them by the other possible pair of symmetrical edges.

Proof. $\bar{I} \cap R$ necessarily equals $(n-1, n)$. Let $Y=\operatorname{Pan}(I)$. There are two possibilities for $\bar{Y} \cap R$ : it equals either $\{(n-2, n),(n-1, n),(n-1, n+1)\}$ or $\{(n-1, n)\}$, depending on whether or not $\bar{I}$ has any entries on the $(n-1)$-th row (or equivalently the $n$-th column). The corresponding values of $\widehat{Y} \cap R$ are $\{(n-2, n),(n-1, n+1)\}$ and $\emptyset$.

Now consider applying $\psi_{C_{n-1}}$ to $\widehat{X}$ and $\widehat{Y}$. Suppose first that we are in the case that $\widehat{Y} \cap R=\emptyset$. This means that the $(n-1)$-th row is empty in $\bar{I}$, so in $\widehat{I}$, both $R$ and the row below $R$ are empty. We have seen already that the fact that $R$ is empty means that there are no edges between vertices numbered at most $n-1$ and those numbered at least $n$. A similar argument shows that the absence of roots in the $(n-1)$-th row implies that the vertices numbered at most $n-2$ are connected to other vertices in that set. It follows that $(n-1)^{(0)}$ and $(n-1)^{(1)}$ are connected in $\psi_{A_{2 n-3}}(\widehat{I})$. By symmetry, $n^{(0)}$ and $n^{(1)}$ are also.

In determining $\psi_{C_{n-1}}(\widehat{X}), n^{(0)}$ gets the label $n-1$. In determining $\psi_{C_{n-1}}(\widehat{Y})$, the label $n-1$ goes to $(n-1)^{(1)}$, the symmetrically opposite vertex. We know that $\psi_{C_{n-1}}(\widehat{Y})$ has no edges connecting vertices $\leq n-1$ with those $\geq n$, so the result of adding the $(n-1)$-th edge is to complete the matchings among the vertices $\leq n-1$. It follows that when we evaluate $\psi_{C_{n-1}}(\widehat{X})$ instead, vertex $n^{(0)}$ will necessarily be connected to the same vertex as $(n-1)^{(1)}$ was in $\psi_{C_{n-1}}(\widehat{Y})$. This means that, while $n^{(0)}$ and $(n-2)^{(1)}$ are connected in $\psi_{C_{n-1}}(\widehat{X})$, we have that $n^{(0)}$ and $(n+1)^{(0)}$ are connected in $\psi_{C_{n-1}}(\widehat{Y})$, establishing the claim.

Now consider the case that $\widehat{Y} \cap R=\{(n-2, n),(n-1, n+1)\}$. In determining $\psi_{C_{n-1}}(\widehat{Y})$, we have that $n^{(0)}$ receives label $n-2$ and $(n+1)^{(0)}$ receives label $n-1$. Since $\widehat{X}$ and $\widehat{Y}$ only differ inside $R$, we have that the $(n-2)$-th column is empty in $\widehat{X}$, so $(n-2)^{(1)}$ receives the $n-2$ label; and we also have that $n^{(0)}$ receives the label $n-1$.

Let us write $b$ for the vertex joined in $n^{(0)}$ in $\widehat{X}$, and $a$ for the vertex joined in $(n-2)^{(1)}$ in $\widehat{X}$. Note that in $\widehat{X}$, there are no edges between $H$ and $H^{c}$, so, prior to the $(n-2)$-th edge being drawn, the four available vertices in $H$ are $(n-2)^{(1)}, a, b, n^{(0)}$ (in clockwise order). 
Now consider what happens when we evaluate $\psi_{C_{n-1}}(\widehat{Y})$. When adding the $(n-2)$-th edge, we connect $n^{(0)}$ to the next available vertex counterclockwise from it, which is $b$. Next, we connect to $(n+1)^{(0)}$ the next available vertex counterclockwise from it, which is $a$.

The result is that $n^{(0)}$ is attached to the same vertex in $\widehat{X}$ and $\widehat{Y}$, but the vertex attached to $(n+1)^{(0)}$ in $\widehat{Y}$ is attached to $(n-2)^{(1)}$ in $\widehat{X}$. This suffices to establish the claim.

The final case of the proposition now follows, because the only edges between $H$ and $H^{c}$ in $\psi_{C_{n-1}}(\widehat{\operatorname{Pan}(I)})$ are the new edges identified above, whose four end-vertices are the result of rotating clockwise the four degree zero vertices of $\psi_{D_{n} / \delta}(I)$.

In order to show the compatibility between $\psi_{D_{n}}$ and Panyushev complementation, we must study the relationship between $s(I)$ and $s(\operatorname{Pan}(I))$. It is straightforward to check that $s(I)$ and $s(\operatorname{Pan}(I))$ are the same iff $I$ contains a root supported over vertex $n-2$ but neither $n-1$ nor $n$. This is equivalent to saying that $\widehat{I}$ includes some root $(j, n-1)$ (i.e., a root on the row just below $R$ ). This can also be described in terms of $\psi_{D_{n}}(I)$, as in the lemma below.

Lemma 3.18. For $I$ an $A_{2 n-3}$-antichain, $I$ contains a root $(j, n-1)$ iff $\psi_{D_{n}}(I)$ contains an edge joining $n-1$ to $k$ with $k$ in $\left\{(n-3)^{(1)}, \ldots, 1^{(1)}, 1^{(0)}, \ldots,(n-2)^{(0)}\right\}$.

Proof. If $I$ has such a root, then the $j$-th edge which is added will be an edge joining $n-1$ to such a $k$. (Since $j \leq n-2$, at the $j$-th step, at least one of the vertices in $\left\{(n-3)^{(1)}, \ldots,(n-2)^{(0)}\right\}$ will be available. $)$

On the other hand, if $\psi_{D_{n}}(I)$ contains such an edge with $k=k^{(0)}$, the only possibility is that there was a root $(j, n-1)$ in $I$. If $k=k^{(1)}$, then an edge from $k$ could have been added at the $k$-th step, but this edge would not have been joining $k^{(1)}$ to $(n-1)^{(0)}$ as there would have been an available vertex with a smaller label.

We say that a vertex is the clockwise end of an edge if the vertex is not degree zero, and the vertex to which it is attached is closer to it counterclockwise than clockwise.

Lemma 3.19. $s(I)=s(\operatorname{Pan}(I))$ iff $(n-1)^{(0)}$ is on the clockwise end of an edge in $\psi_{D_{n} / \delta}(I)$.

Proof. It follows from the previous lemma that $s(I)=s(\operatorname{Pan}(I))$ iff $(n-1)^{(0)}$ is attached to some $k$ in $\left\{(n-3)^{(1)}, \ldots,(n-2)^{(0)}\right\}$ in $\psi_{A_{2 n-3}}(\widehat{I})$.

Suppose $(n-1)^{(0)}$ is attached to some $k$ in $\left\{(n-3)^{(1)}, \ldots,(n-2)^{(0)}\right\}$ in $\psi_{A_{2 n-3}}(\widehat{I})$. Observe that $(n-1)^{(0)}$ cannot be degree zero in $\psi_{D_{n} / \delta}(I)$, because the edge from $(n-1)^{(0)}$ to $k$ is entirely within $H$. Therefore $(n-1)^{(0)}$ is on the clockwise end of its edge.

Conversely, if $(n-1)^{(0)}$ is on the clockwise end of an edge in $\psi_{D_{n} / \delta}(I)$, either it is attached to $k$ in $\left\{(n-3)^{(1)}, \ldots,(n-2)^{(0)}\right\}$, or else it is attached to $(n-1)^{(1)}$. In fact, though, it cannot be attached to $(n-1)^{(1)}$ in $\psi_{D_{n} / \delta}$. If it were the case that $(n-1)^{(0)}$ and $(n-1)^{(1)}$ were attached in $\psi_{A_{2 n-3}}(\widehat{I})$, this edge would have been removed in $\psi_{D_{n} / \delta}(I)$. Thus $s(I)=s(\operatorname{Pan}(I))$. 
We are now ready to prove the following result:

Lemma 3.20. For $I \in \mathrm{NN}\left(D_{n}\right)$, we have that $\psi_{D_{n}}(\operatorname{Pan}(I))=\operatorname{Krew}\left(\psi_{D_{n}}(I)\right)$.

Proof. By Proposition 3.15, we know that $\psi_{D_{n} / \delta}(\operatorname{Pan}(I))=\operatorname{Krew}\left(\psi_{D_{n} / \delta}(I)\right)$. If $I$ lies in a singleton $\delta$-orbit, this is sufficient.

Now suppose $I$ lies in a doubleton $\delta$-orbit. By Proposition 3.15, we know that $\operatorname{Krew}\left(\psi_{D_{n}}(I)\right)$ and $\psi_{D_{n} / \delta}(\operatorname{Pan}(I))$ differ, if at all, only in the way that the singleton vertices are connected.

Let $v$ be a singleton vertex in $\psi_{D_{n} / \delta}(I)$. We know that $\operatorname{Krew}(v)$ is a singleton vertex in $\operatorname{Pan}(I)$. In $\psi_{D_{n}}(I)$, suppose that $v$ is connected to $i$. We then see that $\operatorname{Krew}(v)$ is connected to $i+1$, since the last two terms in the formula cancel each other out by Lemma 3.19 .

3.4. Exceptional types. As for noncrossing partitions in Section 2.5, the exceptional types - as we consider only crystallographic reflection groups, this includes for now the dihedral group $G_{2}$ - were verified using a computer.

\section{Parabolic induction in the Classical types}

In this section, we define the notion of parabolic induction for a collection of maps from $\mathrm{NN}(W)$ to $\mathcal{T}_{W}$, for $W$ a reflection group of classical type, and we show that the previously defined bijections $\psi_{W}$ satisfy this notion of parabolic induction. Further, we show that they are uniquely characterized by this property together with their compatibility with Panyushev complementation and rotation.

4.1. Type $A_{n-1}$. First, consider the case of $W=A_{n-1}$. Pick $i$, with $1 \leq i \leq$ $n-1$. Removing the node $i$ from the Dynkin diagram, we obtain two Dynkin diagrams, of types $A_{i-1}$ and $A_{n-1-i}$. Given noncrossing handshake configurations $U \in \mathcal{T}_{A_{i-1}}$ and $V \in \mathcal{T}_{A_{n-1-i}}$, we can assemble them into a single noncrossing handshake configuration $U * V$ of type $A_{n-1}$, by adding $i$ to the labels of the vertices of $V$. (In order for this to work if $i=1$ or $i=n-1$, we define the unique noncrossing handshake configuration associated to type $A_{0}$ to consist of two vertices, numbered $1^{(0)}$ and $1^{(1)}$, connected by an edge.)

Suppose that $I \in \mathrm{NN}\left(A_{n-1}\right)$ does not have $\alpha_{i}$ in its support. We can then write $I$ as a union of $I_{1}$ supported over a subset of $\alpha_{1}, \ldots, \alpha_{i-1}$, and $I_{2}$ supported over a subset of $\alpha_{i+1}, \ldots, \alpha_{n-1}$.

We say that a collection of maps $F_{A_{n-1}}: \mathrm{NN}\left(A_{n-1}\right) \longrightarrow \mathcal{T}_{A_{n-1}}$ satisfies parabolic induction if, whenever $I \in \mathrm{NN}\left(A_{n-1}\right)$ satisfies that the simple root $\alpha_{i}$ is not in the support of $I$, then

$$
F_{A_{n-1}}(I)=F_{A_{i-1}}\left(I_{1}\right) * F_{A_{n-1-i}}\left(I_{2}\right) .
$$

Proposition 4.1. The maps $\psi_{A_{n-1}}$ satisfy parabolic induction.

Proof. This is an immediate corollary of Proposition 3.3 .

4.2. Type $C_{n}$. Similarly, if we remove a simple root $\alpha_{i}$ from a $C_{n}$ Dynkin diagram, we obtain a diagram of type $A_{i-1}$ and one of type $C_{n-i}$. For convenience, we use 
$C_{1}$ as a pseudonym for $A_{1}$ here. In particular, the noncrossing handshake configurations of type $C_{1}$ are just the noncrossing handshake configurations of type $A_{1}$. By convention, the empty diagram is the unique noncrossing handshake configuration of type $C_{0}$.

Given a noncrossing handshake configuration of type $U \in \mathcal{T}_{A_{i-1}}$ and $V \in \mathcal{T}_{C_{n-i}}$, define $U * V$ to consist of:

- $U$,

- $V$ with its labels increased by $i$,

- $U$ with each label $j$ replaced by $2 n+1-j$, and superscripts (0) and (1) interchanged.

Again, if $I \in \mathrm{NN}\left(C_{n}\right)$ and $\alpha_{i}$ is not in the support of $I$, we can divide $I$ into antichains $I_{1}$ and $I_{2}$. A collection of maps $F_{W}: \mathrm{NN}(W) \rightarrow \mathcal{T}_{W}$ for $W$ of type $A$ or $C$ is said to satisfy parabolic induction if the collection $F_{A_{n}}$ satisfies type $A$ parabolic induction and for $I \in \mathrm{NN}\left(C_{n}\right)$, whenever $\alpha_{i}$ is not in the support of $I$, we have

$$
F_{C_{n}}(I)=F_{A_{i-1}}\left(I_{1}\right) * F_{C_{n-i}}\left(I_{2}\right) .
$$

We have the following corollary of the previous proposition:

Corollary 4.2. The maps $\psi_{A_{n}}, \psi_{C_{n}}$ satisfy parabolic induction.

4.3. Type $D_{n}$. If we remove a simple root $\alpha_{i}$ from a Dynkin diagram of type $D_{n}$, for $i \neq n-1, n$ (the two antennae), then we obtain a Dynkin diagram of type $A_{i-1}$ and a Dynkin diagram of type $D_{n-i}$. Given two noncrossing handshake configurations $U \in \mathcal{T}_{A_{i-1}}$ and $V \in \mathcal{T}_{D_{n-i}}$, we write $U * V$ for the diagram consisting of:

- $U$,

- $V$ with its labels increased by $i$ (including the central ones, where the increase is taken modulo 4),

- The diagram $U$ with label $j$ replaced by $2 n-1-j$, and the superscripts (0) and (1) interchanged.

(We let $D_{2}$ refer to the reducible root system consisting of two orthogonal simple roots and their negatives, and let $D_{3}=A_{3}$. We interpret "noncrossing handshake configuration of type $D_{n}$ " for $n=2,3$, using the type $D$ definition of noncrossing handshake configuration.)

If we remove a simple root $\alpha_{i}$ from a Dynkin diagram of type $D_{n}$, where $i=n-1$ or $n$, then we obtain a Dynkin diagram of type $A_{n-1}$. We will define a pair of maps Ind $_{i}: \mathcal{T}_{A_{n-1}} \rightarrow \mathcal{T}_{D_{n}}$, as follows.

$\operatorname{Ind}_{n}(U)$ is defined to consist of the type $A$ diagram, with vertices $n^{(0)}$ and $n^{(1)}$ moved to the center and renamed $n$ and $n+1$, together with the 180 degree rotation of this diagram. This is a type $D_{n}$ noncrossing handshake configuration by Lemma 3.13 .

$\operatorname{Ind}_{n-1}(U)$ is obtained by adding 2 to each of the labels of the central vertices of $\operatorname{Ind}_{n}(U)$.

Again, if $1 \leq i \leq n-2$, and $I \in \mathrm{NN}\left(D_{n}\right)$ does not have $\alpha_{i}$ in its support, we can define $I_{1} \in \mathrm{NN}\left(A_{i-1}\right)$ and $I_{2} \in \mathrm{NN}\left(D_{n-i}\right)$. If $i=n-1, n$, and $I$ does not have $\alpha_{i}$ in its support, we can simply view $I$ as an antichain in $\mathrm{NN}\left(A_{n-1}\right)$. A collection of maps $F_{W}: \mathrm{NN}(W) \longrightarrow \mathcal{T}_{W}$ for $W=A_{n}, D_{n}$ is said to satisfy parabolic induction 
if the collection $F_{A_{n}}$ satisfies type $A$ parabolic induction, and:

(i) for $1 \leq i \leq n-2$, if $I \in \mathrm{NN}\left(D_{n}\right)$ does not have $\alpha_{i}$ in its support, then

$$
F_{D_{n}}(I)=F_{A_{i-1}}\left(I_{1}\right) * F_{D_{n-i}}\left(I_{2}\right),
$$

and

(ii) for $i=n-1, n$, if $I \in \mathrm{NN}\left(D_{n}\right)$ does not have $\alpha_{i}$ in its support, then

$$
F_{D_{n}}(I)=\operatorname{Ind}_{i}\left(F_{A_{n-1}}(I)\right) \text {. }
$$

Proposition 4.3. The maps $\psi_{D_{n}}, \psi_{A_{n}}$ satisfy parabolic induction.

Proof. Condition (i) follows as in the previous cases. For condition (ii), we divide into cases.

$I \in \mathrm{NN}\left(D_{n}\right)$ has neither $\alpha_{n}$ nor $\alpha_{n-1}$ in its support. In this case, $\bar{I}$ does not intersect $R$. The result in this case follows as in type $C_{n-1}$.

$I \in \mathrm{NN}\left(D_{n}\right)$ has exactly one of $\alpha_{n}, \alpha_{n-1}$ in its support. In this case, $\bar{I} \cap R$ consists of either one root $(n-1, n)$ or two roots $(j, n)$ and $(n-1,2 n-1-j)$. It follows that $\widehat{I} \cap R$ consists of either zero roots or one root.

In the former case, in the type $A_{2 n-3}$ noncrossing handshake configuration associated to $\widehat{I}$, there are no edges from vertices with labels at most $n-1$ to those with labels at least $n$. It follows that the innermost edges from $H$ to $H^{c}$ are connected to $n^{(0)}$ and to $(n-1)^{(1)}$, and thus that in the $D_{n} / \delta$ noncrossing handshake configuration, $(n-1)^{(1)}$ is a singleton vertex. The other singleton vertex with label at most $n-1$, call it $a$, is the one that is connected to $(n-1)^{(1)}$ in the type $A_{2 n-3}$ noncrossing handshake configuration. Now, suppose $I$ is supported over $\alpha_{n-1}$, so $s(I)=0$. We deduce that $(n-1)^{(1)}$ is attached to $n-(2 n-3)+2(n-1)=n+1$. On the other hand, if $I$ is supported over $\alpha_{n},(n-1)^{(1)}$ is attached to $(n+1)+2$.

Now consider the calculation of $\psi_{A_{n-1}}(I)$. Up to the $(n-1)$-th step, the same thing happens. At the $(n-1)$-th step, there now is an entry in the $(n-1)$-th column (namely, $(n-1, n)$ ), so we mark $n^{(0)}$ with label $n-1$, and thus on turn $n-1$, we connect $n^{(0)}$ to the nearest available entry, which must be $a$, since it and $(n-1)^{(1)}$ are the only unmatched vertices on the left-hand side. On the final step, we join $n^{(1)}$ and $(n-1)^{(1)}$. We see that $\psi_{D_{n}}(I)=\operatorname{Ind}_{n-s(I)}\left(\psi_{A_{n-1}}(I)\right)$.

Next, consider the case that $\widehat{I} \cap R$ has one root in its support, say $(i, 2 n+1-i)$. Consider the calculation of $\psi_{A_{2 n-3}}(\widehat{I})$ and of $\psi_{A_{n-1}}(I)$ in parallel. The same thing happens in both up to the $i$-th step. On the $i$-th step of the $A_{n-1}$ calculation, the label $i$ goes onto the node $n^{(0)}$, so we connect $n^{(0)}$ to $(n-1)^{(0)}$ at this point, while for the $A_{2 n-3}$ calculation, we connect $(2 n+1-i)^{(0)}$ to $(2 n-i)^{(0)}$. From here on, the calculations run the same way up to and through the $(n-1)$-th step. In both calculations, there is no entry in the $(n-1)$-th column, so we connect $(n-1)^{(1)}$ to some entry on the left-hand side. After this step, in the calculation of $\psi_{A_{2 n-3}}(\widehat{I})$, there are two remaining unmatched vertices whose labels are at most $n-1$. One of them is $(n-1)^{(0)}$, while we call the other one $a$. It follows that the four vertices in $H$ which will eventually be matched to vertices in $H^{c}$ are, in clockwise order, the vertex attached to $(n-1)^{(1)}, a,(n-1)^{(0)}$, and (by symmetry) $n^{(0)}$. The two innermost edges are therefore the ones attached to $a$ and $(n-1)^{(0)}$. It follows that we will connect $a$ and $(n-1)^{(0)}$ to the internal vertices, and $(n-1)^{(0)}$ will be connected to $n$ if $s(I)=0$ and $n+2$ if $s(I)=1$.

On the $n$-th step of the $\psi_{A_{n-1}}(I)$ calculation, we connect $n^{(1)}$ to the only available vertex, $a$. We therefore see that $\operatorname{Ind}_{n-s(I)}\left(\psi_{A_{n-1}}(I)\right)=\psi_{D_{n}}(I)$, as desired. 
4.4. Uniqueness of $\psi$ in the classical types. Finally, we show that parabolic induction determines $\psi$ uniquely in the classical cases. In this section, we show that:

Theorem 4.4. The only collection of bijections $F_{W}: \mathrm{NN}(W) \rightarrow \mathcal{T}_{W}$, for $W$ running over all classical irreducible reflection groups, that satisfy:

(i) $F_{W} \circ$ Pan $=$ Krew $\circ F_{W}$, and

(ii) classical parabolic induction, as defined previously,

are the maps $F_{W}=\psi_{W}$.

Proof. We have already shown that the maps $\psi_{W}$ do satisfy the two properties mentioned in the theorem; we need only show that these two properties are sufficient to characterize these functions uniquely.

By property (i), it suffices to know that, for any Pan orbit in $\mathrm{NN}(W)$, there is some antichain to which some parabolic induction applies. Expressed in those terms, it is not obvious that this is true. However, thanks to the bijections $\psi_{W}$, it is sufficient to show that for any Krew orbit in $\mathcal{T}_{W}$, there is a noncrossing handshake configuration which could have arisen by parabolic induction. This is quite clear. Let $T$ be a noncrossing handshake configuration of type $W$. Pick some edge joining two external vertices. After applying a suitable power of Krew to $T$, the chosen edge connects $i^{(0)}$ to $i^{(1)}$. In type $A_{n-1}$, this implies that $T$ comes from a parabolic induction $A_{i-1} * A_{1} * A_{n-i}$, where at most one of these is zero. A completely similar approach works in type $C$ or $D$, except in the case of $D_{2}$, since in that case there is a Krew orbit with no edge connecting a pair of external vertices. However, it is easy to check that both the elements of that orbit arise via Ind. This completes the proof.

\section{A UNIFORM, RECURSIVE BIJECTION}

In this section, we prove the Main Theorem. We begin with the classical types.

Let $W$ be a reflection group of classical type, and let $L, R$ be a bipartition of its simple roots. For each of the three classical families, we define a certain bijection $\phi_{(L, R)}: \mathcal{T}_{W} \rightarrow \mathrm{NC}\left(W, c_{L} c_{R}\right)$, which will be a mild variant of $\phi_{W}$ as defined in Section 2, Then we define $\Lambda_{(L, R)}: \mathrm{NN}(W) \rightarrow \mathrm{NC}\left(W, c_{L} c_{R}\right)$ by setting $\Lambda_{(L, R)}(I)=\phi_{(L, R)} \psi_{W}(I)$. We then check that this bijection satisfies conditions (i)-(iii) of the Main Theorem.

Next, we show that the Main Definition yields a well-defined map (using results from the previous section in the classical types, and a computer check for the exceptional types). We also show that a map satisfying conditions (i)-(iii) of the Main Theorem must be the map defined by the Main Definition. Thus, the bijection $\Lambda_{(L, R)}$ described above must be the map defined by the Main Definition. This completes the proof of the Main Theorem in the classical types. Finally, we check (with a computer) that the maps defined by the Main Definition also satisfy the Main Theorem in the exceptional types, which completes the proof of the Main Theorem.

5.1. Type $A_{n-1}$. Let $\left\{s_{1}, \ldots, s_{n-1}\right\}$ with $s_{i}=(i, i+1)$ be the generators in type $A_{n-1}$, and let $c_{L} c_{R}$ be a bipartite Coxeter element. As mentioned in Remark 1, we can cyclically label the vertices of the noncrossing handshake configurations in $\mathcal{T}_{n}$ 
by the Coxeter element $c_{L} c_{R}$. If $s_{1} \in L$, the cyclic labelling for $\phi_{(L, R)}$ is given by

$$
2^{(0)}, 2^{(1)}, 4^{(0)}, 4^{(1)}, \ldots, 3^{(0)}, 3^{(1)}, 1^{(0)}, 1^{(1)},
$$

and if $s_{1} \in R$, the cyclic labelling for $\phi_{(L, R)}$ is given by

$$
1^{(1)}, 3^{(0)}, 3^{(1)}, \ldots, 4^{(0)}, 4^{(1)}, 2^{(0)}, 2^{(1)}, 1^{(0)} .
$$

Theorem 5.1. The bijections

$$
\begin{aligned}
& \Lambda_{A_{n-1},(L, R)}: \mathrm{NN}\left(A_{n-1}\right) \stackrel{\sim}{\longrightarrow} \mathrm{NC}\left(A_{n-1}, c_{L} c_{R}\right), \\
& \Lambda_{A_{n-1},(R, L)}: \mathrm{NN}\left(A_{n-1}\right) \stackrel{\sim}{\longrightarrow} \mathrm{NC}\left(A_{n-1}, c_{R} c_{L}\right)
\end{aligned}
$$

satisfy conditions (i)-(iii) of the Main Theorem in type A.

Proof. We will only check the first statement; the proof of the second is identical. The initial condition is easily verified. The Pan $=$ Krew condition follows from the facts that $\psi_{A_{n-1}} \circ$ Pan $=$ Krew $\circ \psi_{A_{n-1}}$ and $\phi_{(L, R)} \circ$ Krew $=$ Krew $\circ \phi_{(L, R)}$.

As we have proved the parabolic recursion for $\psi_{A_{n-1}}$ in the previous section, it is left to prove the analogous statement for $\phi_{(L, R)}$. Let $T \in \mathcal{T}_{n}$ be a noncrossing handshake configuration such that $T_{1}=\left\{i^{(0)}, i^{(1)}: 1 \leq i \leq k\right\}$ and $T_{2}=\left\{i^{(0)}, i^{(1)}: k<i \leq n\right\}$ define submatchings of $T$ with vertices being labelled as in Proposition 3.3. We have to show that

$$
\phi_{(L, R)}(T)=\left\{\begin{aligned}
\phi_{\left(L_{1}, R_{1}\right)}\left(T_{1}\right) \phi_{\left(L_{2}, R_{2}\right)}\left(T_{2}\right) & \text { if } s_{k} \in R, \\
s_{k} \phi_{\left(L_{1}, R_{1}\right)}\left(T_{1}\right) \phi_{\left(R_{2}, L_{2}\right)}\left(T_{2}\right) & \text { if } s_{k} \in L,
\end{aligned}\right.
$$

where $L_{1 / 2}=L \cap S_{1 / 2}$ and $R_{1 / 2}=R \cap S_{1 / 2}$ with $S_{1}=\left\{s_{1}, \ldots, s_{k-1}\right\}$ and $S_{2}=$ $\left\{s_{k+1}, \ldots, s_{n-1}\right\}$. This results in 4 different cases.

Case 1: $s_{1} \in L, s_{k} \in R$. In this case, the labelling is as in (5) and $k$ is even. The statement follows as the labelling of $T_{1}$ is given by

$$
2^{(0)}, 2^{(1)}, \ldots, k^{(0)}, k^{(1)},(k-1)^{(0)},(k-1)^{(1)}, \ldots, 1^{(0)}, 1^{(1)},
$$

and the labelling of $T_{2}$ is given by the remaining labels. These are exactly the labellings obtained as well for $\phi_{\left(L_{1}, R_{1}\right)}\left(T_{1}\right)$ and $\phi_{\left(L_{2}, R_{2}\right)}\left(T_{2}\right)$.

Case 2: $s_{1} \in L, s_{k} \in L$. In this case, the labelling is as in (5) and $k$ is odd. The labelling of $T_{1}$ is now given by

$$
2^{(0)}, 2^{(1)}, \ldots,(k+1)^{(0)}, k^{(1)}, \ldots, 1^{(0)}, 1^{(1)},
$$

and the labelling of $T_{2}$ is given by the remaining labels. It is a straightforward check that this differs from the labelling for $\phi_{\left(L_{1}, R_{1}\right)}\left(T_{1}\right)$ and for $\phi_{\left(R_{2}, L_{2}\right)}\left(T_{2}\right)$ by having the labels $(k+1)^{(0)}$ and $k^{(0)}$ interchanged. This corresponds exactly to the additional factor $s_{k}$.

The remaining two cases for $s_{1} \in R$ are solved in the analogous way.

5.2. Type $C_{n}$. As above, the bipartite Coxeter elements in type $C_{n}$ can be obtained from bipartite Coxeter elements in type $A_{2 n-1}$, where $-i$ and $2 n+1-i$ are identified. The bijection in type $C$ then follows as a simple corollary from the construction in type $A$. 
Corollary 5.2. The bijections

$$
\begin{aligned}
& \Lambda_{C_{n},(L, R)}: \mathrm{NN}\left(C_{n}\right) \stackrel{\sim}{\longrightarrow} \mathrm{NC}\left(C_{n}, c_{L} c_{R}\right), \\
& \Lambda_{C_{n},(R, L)}: \mathrm{NN}\left(C_{n}\right) \stackrel{\sim}{\longrightarrow} \mathrm{NC}\left(C_{n}, c_{R} c_{L}\right)
\end{aligned}
$$

satisfy conditions (i)-(iii) of the Main Theorem in type $C$.

5.3. Type $D$. Exactly the same argument as in type $A_{n-1}$ applies to the bipartite Coxeter elements in type $D_{n}$. Those are obtained from the bipartite Coxeter element in type $A_{n-1}$ by adding $s_{n}=(n-1,-n)$ to $L$ if $n$ is even and to $R$ if $n$ is odd. E.g., in type $D_{4}$, we obtain the cyclic labelling on the outer circle for $c_{L} c_{R}$ given by

$$
2^{(0)}, 2^{(1)},-3^{(0)},-3^{(1)},-1^{(0)},-1^{(1)},-2^{(0)},-2^{(1)}, 3^{(0)}, 3^{(1)}, 1^{(0)}, 1^{(1)},
$$

and the inner circle labelling by $4^{(0)}, 4^{(1)},-4^{(0)},-4^{(1)}$. The labellings for $c_{R} c_{L}$ are again given by reflecting the labels at the diagonal through $1^{(1)}$.

Corollary 5.3. The bijections

$$
\begin{aligned}
& \Lambda_{D_{n},(L, R)}: \mathrm{NN}\left(D_{n}\right) \stackrel{\sim}{\longrightarrow} \mathrm{NC}\left(D_{n}, c_{L} c_{R}\right), \\
& \Lambda_{D_{n},(R, L)}: \mathrm{NN}\left(D_{n}\right) \stackrel{\sim}{\longrightarrow} \mathrm{NC}\left(D_{n}, c_{R} c_{L}\right)
\end{aligned}
$$

satisfy conditions (i)-(iii) of the Main Theorem in type D.

Proof. The proof follows the same lines as the proof in type $A$, with the additional check for the cases in which $s_{n-1}$ or $s_{n}$ are not contained in the support of an antichain $I \in \mathrm{NN}\left(D_{n}\right)$. Using Theorem 4.4 in type $D_{n}$, this check is straightforward.

5.4. The Main Definition and the Main Theorem. We now establish that the Main Definition does yield a well-defined map. As we remarked in the Introduction, this requires precisely that we show that in every Pan-orbit of $\mathrm{NN}(W)$, there is an antichain which does not have full support. In the classical types, this was established in the proof of Theorem 4.4, while in the exceptional types, it is easily verified with a computer.

We will denote the map defined by the Main Definition by $\Theta_{(L, R)}$. (We prefer this to the simpler notation, $\Theta_{W}$, which we used in the Introduction, because it is important to keep track of the bipartition of the simple roots.)

Next, we check that any map satisfying conditions (i)-(iii) of the Main Theorem must coincide with $\Theta_{(L, R)}$. Let $\Psi_{(L, R)}$ be a map satisfying conditions (i)-(iii) of the Main Theorem. We prove by induction on the rank of $W$ that $\Psi_{(L, R)}=\Theta_{(L, R)}$.

Let $J$ be an antichain in $\mathrm{NN}(W)$. Let $k$ be minimal such that $I=\operatorname{Pan}^{k}(J)$ has less than full support. Now $\Theta_{(L, R)}(I)=\Psi_{(L, R)}(I)$ by comparing step (ii) from the Main Definition with the parabolic induction property in the Main Theorem. Finally $\Theta_{(L, R)}(J)=\mathrm{Krew}^{-k} \Theta_{(L, R)}(I)=\mathrm{Krew}^{-k} \Psi_{(L, R)}(I)$, and by Pan $=\mathrm{Krew}$, this equals $\Psi_{(L, R)}(J)$, as desired.

This finishes the proof that any map satisfying properties (i)-(iii) of the Main Theorem must be the map defined by the Main Definition. Since we have already established that the maps $\Lambda_{(L, R)}$ satisfy conditions (i)-(iii) of the Main Theorem, we must have that $\Lambda_{(L, R)}=\Theta_{(L, R)}$ in the classical types, which finishes the proof of the Main Theorem in these types. For the exceptional types, it is relatively easy to check with a computer that the maps defined by the Main Definition really do 
satisfy conditions (i)-(iii) of the Main Theorem, and the proof of the Main Theorem is completed.

\section{A proof of the Panyushev Conjectures}

In this final section of the paper, we will use combinatorial results described in the previous sections to prove the Panyushev Conjectures. The first proposition follows directly from the uniform description of the bijection.

Proposition 6.1. Part (i) of the Panyushev Conjectures holds: $\operatorname{Pan}^{2 h}$ is the identity map on $\mathrm{NN}(W)$.

Proof. This follows from the connection to the Kreweras complementation and the fact that $\mathrm{Krew}^{2 h}$ is the identity map on $\mathrm{NC}(W)$.

For all remaining proofs, we use the combinatorics obtained for the classical types, and computer checks for the exceptionals. To prove (ii) of the Panyushev Conjectures, it remains to show that $\mathrm{Krew}^{h}$ acts on $\mathrm{NN}(W)$ by the involution induced by $-\omega_{0}$. Thus, we have two cases, depending on how $-\omega_{0}$ acts on Dynkin diagrams:

(iia) $\mathrm{Krew}^{h}$ acts trivially on $\Phi$ in types $C_{n}, D_{2 n}, F_{4}, E_{7}$, and $E_{8}$.

(iib) In the remaining types $A_{n-1}, D_{2 n+1}$, and $E_{6}$, the action of $\mathrm{Krew}^{h}$ is induced by the involution on the Dynkin diagram (called $\delta$ in types $A$ and $D$ ).

Proof of part (ii) of the Panyushev Conjectures. In types $A$ and $C$, (iia) and (iib) follow from the symmetry property of noncrossing handshake configurations (see Lemma 3.5). In type $D$, (iia) and (iib) follow from the facts that rotating a type $D_{n} / \delta$ noncrossing handshake configuration by $2(n-1)$ steps yields the same configuration, but to obtain the same $D_{n}$ noncrossing handshake configuration, it is also necessary to ensure that the number of rotations applied yields a half-turn of the 4 inner vertices. Type $E_{6}$ was checked with a computer. The statements for the remaining exceptional types can be verified using the orbit lengths found in Section 2.5.

Proof of part (iii) of the Panyushev Conjectures. First we consider type $A_{n-1}$. Pick a noncrossing handshake configuration $X$, and consider

$$
X, \operatorname{Krew}(X), \ldots, \operatorname{Krew}^{2 n-1}(X) .
$$

Each edge $e$ in $X$ appears (rotated) in each of these noncrossing handshake configurations, and we see that some endpoint of $e$ is labelled with (0) and marked in $n-1$ of these noncrossing handshake configurations. In a given noncrossing handshake configuration, the number of vertices labelled with (0) and marked is exactly the number of positive roots in the corresponding antichain, so we see that the total number of positive roots in the antichains corresponding to these $2 n$ noncrossing handshake configurations is $n-1$ times the number of edges, which is $n$. It follows that the average number of positive roots in the corresponding Pan orbit is $(n-1) / 2$.

The easiest way to prove the result for type $C_{n}$ is the following: it is straightforward to check that every second antichain in a Panyushev orbit contains a positive 
root of the form $(i, \bar{i})$. As type $A_{2 n-1}$ folds to the type $C_{n}$, the total number of antichains in an orbit in type $C_{n}$ is given by

$$
\frac{\frac{4 n}{2} \frac{2 n-1}{2}+2 n}{4 n}=\frac{n}{2} \text {. }
$$

Here, the numerator contains $4 n \frac{2 n-1}{2}$, which is the orbit size (without symmetry) times the average number of elements in the orbit in type $A_{2 n-1}$, the division by 2 comes from the folding, and the correction term $2 n$ comes from the centered element in every other orbit which is not folded. The $4 n$ in the denominator is again the size of the orbit. (If we have a $k$-fold symmetry, all three pieces obtain a factor of $1 / k$.) This completes the proof in type $C$.

In type $D$, the situation is again a little more involved. We will work in terms of $D_{n} / \delta$ configurations. There are two different cases, based on whether or not there are four isolated vertices on the outside.

Suppose first that there are not. Each such $D_{n}$ antichain corresponds to a $C_{n-1}$ antichain, and the Panyushev map respects this folding action. Thus, a Panyushev orbit of such $D_{n}$ antichains corresponds to a Panyushev orbit of $C_{n-1}$ antichains; the average number of roots present in these $C_{n-1}$ antichains is $(n-1) / 2$. The $D_{n}$ antichain $I$ corresponding to a $C_{n-1}$ antichain $I^{\prime}$ is just the inverse image of $I^{\prime}$ under the folding map from $\Phi_{D_{n}}$ to $\Phi_{C_{n-1}}$. The number of elements in $I$ equals the number of elements in $I^{\prime}$ plus the number of elements in $I^{\prime}$ whose inverse image consists of two roots; there will be either one or zero such roots in $I^{\prime}$. We observe that there is such a root in $I^{\prime}$ iff $n^{(0)}$ is marked. As we rotate $\psi_{C_{n-1}}\left(I^{\prime}\right)$ through a full rotation, each edge of the configuration is connected to vertex $n^{(0)}$ twice, once at each of its endpoints, and it is easy to see that once we will have $n^{(0)}$ marked, while once it will be unmarked. Thus, the average effect of passing from $I^{\prime}$ to $I$ is to add $\frac{1}{2}$ to the size of the antichains, resulting in an average size of $n / 2$, as desired.

Now suppose that there are four isolated vertices in $\psi_{D_{n} / \delta}(I)$. We consider first the average size of $\hat{I}$ (which, we recall, is an antichain of type $A_{2 n-3}$ ). Recall that, as we consider $\psi_{A_{2 n-3}}(\hat{I}), \psi_{A_{2 n-3}}(\widehat{\operatorname{Pan}(I)}), \ldots$, the effect is to rotate the noncrossing handshake configuration except that there is one pair of edges which, at a certain point, gets switched, and then eventually switches back; in a full rotation $(4 n-4$ steps) this happens twice.

Consider first an edge which is not involved in the switching. It contributes a marked vertex $2 n-3$ times (out of the $4 n-4$ rotations). Now consider the pair of edges that are involved in the switching. One verifies directly that they contribute, together, $4 n-8$ marked vertices. The average size of the antichains $\widehat{I}, \widehat{\operatorname{Pan}(I)}$, etc., is $[(2 n-4)(2 n-3)+(4 n-8)] /(4 n-4)=\left(4 n^{2}-10 n+4\right) /(4 n-4)$.

We next consider the average size of the sets $\bar{I}, \overline{\operatorname{Pan}(I)}$, etc. Each of these contains one more root than the corresponding antichain $\widehat{I}, \widehat{\operatorname{Pan}(I)}$, etc., so the average size of these sets is $\left(4 n^{2}-6 n\right) /(4 n-4)$.

Next we consider the relationship between the size of $\bar{I}$ and the size of $I$. The size of $I$ is $|\bar{I}| / 2$, plus a correction of $\frac{1}{2}$ if $\bar{I}$ has an element on the central diagonal. Over $4 n-4$ rotations, the correction will appear $2 n$ times (i.e., two more than half the time). The reason for this is that, if $I$ is such that $\widehat{I}$ and $\widehat{\operatorname{Pan}(I)} \operatorname{differ}$ by a switch of the edges, then neither of them will have an element on the central diagonal. We see this because of the fact that the switching edges are the most 
internal among those connecting $H$ to $H^{c}$ in $\psi_{A_{2 n-3}}(\widehat{I})$. Now $\widehat{I}$ has no element on the central diagonal iff $\bar{I}$ does have an element on the central diagonal.

It follows that the number of elements in an antichain, averaged over a Pan-orbit, is $\left(4 n^{2}-4 n\right) /(8 n-8)=n / 2$.

\section{ACKNOWLEDGEMENTS}

The authors would like to thank Christian Krattenthaler and Vic Reiner for helpful discussions.

The Main Theorem was verified in the exceptional types with the help of J. Stembridge's coxeter and posets packages for Maple.

The orbit sizes for the Kreweras and the Panyushev complements were calculated using Sage [25].

\section{REFERENCES}

1. D. Armstrong, Generalized noncrossing partitions and combinatorics of Coxeter groups, Mem. Amer. Math. Soc. 202 (2006), no. 949. MR2561274 (2011c:05001)

2. C.A. Athanasiadis, Generalized Catalan numbers, Weyl groups and arrangements of hyperplanes, Bull. London Math. Soc. 36 (2004), 294-302. MR2038717 (2005b:52055)

3. C.A. Athanasiadis and V. Reiner, Noncrossing partitions for the group $D_{n}$, SIAM J. Discrete Math. 18 (2004), 397-417. MR2112514(2006b:06004)

4. Y. Berest, P. Etingof, and V. Ginzburg, Finite dimensional representations of rational Cherednik algebras, Int. Math. Res. Not. 19 (2003), 1053-1088. MR1961261 (2004h:16027)

5. O. Bernardi, Bijective counting of tree-rooted maps and shuffles of parenthesis systems, Electron. J. Combin. 14 (2007), no. 1, R9. MR2285813 (2007m:05125)

6. D. Bessis and V. Reiner, Cyclic sieving of noncrossing partitions for complex reflection groups, Ann. Comb. 15 (2011), 197-222. MR2813511

7. A. E. Brouwer and A. Schrijver, On the period of an operator, defined on antichains, Mathematisch Centrum, Amsterdam, 1974. MR0349497 (50:1990)

8. P. Cellini and P. Papi, ad-nilpotent ideals of a Borel subalgebra II, J. Algebra 258 (2002), 112-121. MR1958899(2004e:17006)

9. W.Y.C. Chen, E.Y.P. Deng, R.R.X. Du, R.P. Stanley, and C.H. Yan, Crossings and nestings of matchings and partitions, Trans. Amer. Math. Soc. 359 (2007), no. 4, 1555-1575. MR 2272140 (2007i:05015)

10. P. Duchet, Sur les hypergraphes invariantes, Discrete Math. 8 (1974), 269-280. MR.0340029 $(49: 4786)$

11. S.-P. Eu and T.-S. Fu, The cyclic sieving phenomenon for faces of generalized cluster complexes, Adv. in Appl. Math. 40 (2008), no. 3, 350-376. MR2402175 (2009f:05268)

12. A. Fink and B.I. Giraldo, A bijection between noncrossing and nonnesting partitions for classical reflection groups, Proceedings of the 21st International Conference on Formal Power Series and Algebraic Combinatorics, DMTCS (2009), 399-412.

13. J. Fürlinger and J. Hofbauer, q-Catalan numbers, J. Combin. Theory Ser. A 40 (1985), no. 2, 248-264. MR814413(87e:05017)

14. M. Haiman, Conjectures on the quotient ring by diagonal invariants, J. Algebraic Combin. 3 (1994), 17-76. MR1256101 (95a:20014)

15. C.E. Heitsch, Combinatorics on plane trees, motivated by RNA secondary structure configurations, preprint.

16. Kreweras complementation and orbits in Catalan lattices, preprint.

17. C. Krattenthaler, Non-crossing partitions on an annulus, in preparation.

18. C. Krattenthaler and T.W. Müller, Cyclic sieving for generalised non-crossing partitions associated to complex reflection groups of exceptional type, preprint, available at arXiv:1001.0028 (2010).

19. G. Kreweras, Sur les partitions non-croisées d'un cycle, Discrete Math. 1 (1972), 333-350. MR0309747(46:8852)

20. D.I. Panyushev, On orbits of antichains of positive roots, European J. Combin. 30 (2009), no. 2, 586-594. MR2489252 (2010c:05145) 
21. V. Reiner, Non-crossing partitions for classical reflection groups, Discrete Math. 177 (1997), 195-222. MR:1483446(99f:06005)

22. V. Reiner, D. Stanton, and D. White, The cyclic sieving phenomenon, J. Combin. Theory Ser. A 108 (2004), 17-50. MR2087303 (2005g:05014)

23. M. Rubey and C. Stump, Crossings and nestings in set partitions of classical types, Electron. J. Combin. 17 (2010), no. 1, R120. MR2729369 (2012c:05042)

24. D. Rush and X.L. Shi, On orbits of order ideals of minuscule posets, preprint, available at arXiv: 1108.5245 (2011).

25. W.A. Stein et al., Sage Mathematics Software (Version 4.6), The Sage Development Team, 2011, http://www . sagemath.org.

26. J. Striker and N. Williams, Promotion and rowmotion, preprint, available at arXiv:1108.1172 (2011).

Department of Mathematics, University of Miami, Coral Gables, Florida 33146

E-mail address: armstrong@math.umiami.edu

LaCiM, Université du Québec À Montréal, Montréal, Québec, Canada

E-mail address: christian.stump@univie.ac.at

Current address: Institut für Algebra, Zahlentheorie und Diskrete Mathematik, Universität Hannover, Germany

Department of Mathematics and Statistics, University of New Brunswick, FrederICTON, NEW BRUnSwick, E3B 5A3, CANADA

E-mail address: hthomas@unb.ca 